\title{
Advanced Methods and Algorithms for Biological Networks Analysis
}

\author{
HANA EL-SAMAD, MEMBER, IEEE, STEPHEN PRAJNA, MEMBER, IEEE, \\ ANTONIS PAPACHRISTODOULOU, MEMBER, IEEE, JOHN DOYLE, MEMBER, IEEE,
} AND MUSTAFA KHAMMASH, SENIOR MEMBER, IEEE

Invited Paper

\begin{abstract}
Modeling and analysis of complex biological networks presents a number of mathematical challenges. For the models to be useful from a biological standpoint, they must be systematically compared with data. Robustness is a key to biological understanding and proper feedback to guide experiments, including both the deterministic stability and performance properties of models in the presence of parametric uncertainties and their stochastic behavior in the presence of noise. In this paper, we present mathematical and algorithmic tools to address such questions for models that may be nonlinear, hybrid, and stochastic. These tools are rooted in solid mathematical theories, primarily from robust control and dynamical systems, but with important recent developments. They also have the potential for great practical relevance, which we explore through a series of biologically motivated examples.

Keywords-Biological networks, model invalidation, robust stability, sum of squares based software tools (SOSTOOLS), stochastic analysis.
\end{abstract}

\section{INTRODUCTION}

Over the past decade, great advances in instrumentation and several crucial biological discoveries have allowed experimental molecular biology to provide detailed descriptions of the components and microscopic processes initiated

\footnotetext{
Manuscript received September 10, 2005; revised December 9, 2005. This work was supported in part by the Army Institute for Collaborative Biotechnologies, in part by the National Science Foundation under Award CCF0326635 (ITR COLLAB: Theory and Software Infrastructure for a Scalable Systems Biology) and Award CCF-0326576, in part by the Air Force Office of Scientific Research (AFOSR) under Award FA9550-05-1-0032, Bio Inspired Networks, and in part by the Boeing Company.

H. El-Samad is with the California Institute for Quantitative Biomedical Research, University of California at San Francisco, San Francisco, CA 94143-2542 USA (e-mail: helsamad@biochem.ucsf.edu).

S. Prajna, A. Papachristodoulou, and J. Doyle are with Control and Dynamical Systems, California Institute of Technology, Pasadena, CA 91125 USA (e-mail: prajna@cds.caltech.edu; antonis@cds.caltech.edu; doyle@cds.caltech.edu).

M. Khammash is with Mechanical and Environmental Engineering, University of California at Santa Barbara, Santa Barbara, CA 93106 USA (e-mail: Khammash@engineering.ucsb.edu).
}

Digital Object Identifier 10.1109/JPROC.2006.871776 by individual molecules within a cell, in addition to high-fidelity accounts of their macroscopic phenotypic effects on cells and organisms. As a consequence, there are increasingly more detailed snapshots of the underlying networks, circuits, and pathways responsible for the basic functionality and robustness of biological systems. A direct result of such snapshots is the realization that the implementation of even the simplest biological networks involves layers of complex nonlinear interactions, time delays, positive and negative feedback, redundancy, and crosstalk. Collectively, this makes the interactions between the elements and the resulting complex dynamics often intractable through intuition alone. Therefore, the mathematical modeling of biological networks has become necessary in linking the known components, the range of dynamical behavior these components can generate, and experimental data [1]-[3]. Once this is accomplished, one can additionally study the effect of perturbations (such as environmental stressors) on these systems. One can also envisage the use of such understanding in assessing the causes of disease (the failure of these regulatory networks) or even the effects of administration of therapeutic drugs [4]-[6].

Biological model building, validation and analysis are nonetheless complicated tasks, and at their core lie some of the most fundamental mathematical challenges of the nascent field of systems biology. Broadly stated, in order to devise reliable and hence useful biological models, one needs to systematically and rigorously connect experimental data to modeling, analysis, and inference, and provide tight feedback to experimentation and modeling throughout. With the appropriate framework, more concise questions can be formulated. Is a model consistent with experimental data which may come from extremely heterogeneous sources? If so, is it robust to additional perturbations that are biologically plausible but as yet untested? What is the most promising experiment to refute a model? Put in natural 
terms (which are typically stochastic, nonlinear, nonequilibrium, hybrid, and so on), such questions are conventionally viewed as computationally intractable. Thus, biologists and engineers alike are often forced to resort to inefficient simulation methods or translate their problem into biologically unnatural terms in order to use available algorithms; hence the necessity for an algorithmic scalable infrastructure that systematically addresses these questions.

In this paper, we report on new contributions to the development of a scalable scientific theory and software infrastructure for complex biological networks. These build on decades of research in the mathematics of controls and dynamical systems, but particularly on recent developments. We specifically provide a promising approach to model validation, robustness, and stochastic reachability analysis, all in the context of two biologically motivated and functionally important systems: the heat-shock (HS) response in E. coli and the lysis/lysogeny decision system in the bacteriophage $\lambda$. Both are among the most familiar and widely studied networks in biology. More specifically, using the HS response as a case study, we study the important problem of model validation/invalidation. In biological modeling, model validation is usually carried out by comparing the model predictions to data. Implicitly underlying this task is the assumption that models can be unambiguously compared with data. In fact, this comparison is in some respects even more computationally challenging than modeling and analysis itself. That is, given a model with a large number of unknown parameters, simulation plus local sensitivity analysis and search can sometimes produce parameter values that fit data or are locally maximally likely to fit. If this fails, however, there may be no short proof that the model is incompatible with the data. Furthermore, with sufficiently complex models and a large number of parameters, it is well known that almost any finite amount of data can be fit.

A closely related problem involves comparing different models of the same system. For example, suppose that a given complex model has been successfully fit to existing data and has passed reasonable invalidation and robustness tests. A common question then is whether the complexity is necessary or whether a simpler model can have essentially the same behavior and robustness. Model invalidation can be used to explore this. Using the HS response in the bacterium E. coli as a test case, we illustrate how such problems can be systematically approached. We specifically invalidate a HS model lacking what we show is a crucial component. As opposed to exhaustive simulations and searches of the parameter space, we use the sum of squares based software tools (SOSTOOLS) to construct a barrier certificate that separates data points from the dynamical behavior generated by the deficient model [7]. Therefore, the deficient model is guaranteed to be inconsistent with the data for all values of the parameters (laying within a plausible set), and thus the missing component is structurally important in the system.

We also consider robust stability analysis of the HS system. Our interest in robust stability of biological systems is in great part motivated by the fact that evaluating the robustness of these models to biochemically plausible parameter variations and other perturbations is emerging as a reliable method for rejecting over-fit models [8]. In fact, an idea that is gaining acceptance with the advent of multiple examples is that a model which is not robust is not likely to be biologically meaningful. In this situation one might suggest that critical pieces of the real system have not been captured. In essence, robustness of a model is a good indicator of its plausibility and, further, robust systems are highly nongeneric and have specialized structures unlikely to arise by fitting incorrect models to data.

Biological networks exhibit extraordinary robustness to common perturbations in their environments, but are also often catastrophically disabled by relatively small but unusual perturbations. For example, the immune system that has an extraordinarily robust capability of recognizing self from foreign entities can cause massive failure of vital organs in response to minuscule (and rare) autoimmune damage that generates self-destructing lymphocytes. Elucidating this ubiquitous "robust yet fragile" nature of biological systems in any particular problem domain is often a key to understanding the origin of network complexity [4], [9]-[11]. Theory and modeling approaches that do not explicitly exploit the highly structured, evolved, and "robust yet fragile" nature of biological systems are hopelessly doomed to be overwhelmed by their sheer complexity. We therefore study the problem of systematic robust stability analysis, again in the context of the HS response and using SOSTOOLS to construct appropriate Lyapunov functions. Although the theory of Lyapunov stability is more than a century old [12], the newer contributions of sum of squares and SOSTOOLS that we discuss provide a relaxation that facilitates the systematic computation of Lyapunov functions [13].

The analysis of the HS system is carried for a deterministic mathematical model that assumes that the interactions between the various cellular components are continuous processes with no uncertainty, while the analysis we present for the bacteriophage $\lambda$ is carried in a more natural stochastic context. Considered as a set of well-stirred biochemical reactions, interactions between the components of biochemical networks are traditionally mathematically approximated by a set of coupled ordinary differential equations, when in fact molecular populations are whole numbers that change by discrete, integer amounts. Furthermore, the occurrence of chemical reactions is by itself a random process subject to thermal fluctuations, and hence stochastic. Whereas discreteness and stochasticity may not be noticeable in "test-tube" size or larger system when the molecular populations of some reactant species are very small, or if the dynamic structure of the system makes it susceptible to noise amplification as is often the case in cellular systems, they can play an important role. Whenever that happens, ordinary differential equations may poorly describe the true behavior of the system. This is further corroborated by physical evidence pointing to purely stochastic phenomena in a number of genetic networks [14]-[18].

These phenomena are particularly interesting in multistable systems where the interaction of large noise intensities 
could, for example, lead to random switching from one cellular state to another [19]. Even in the presence of small noise intensities, switching events can still happen on long time scales. It has been hypothesized that such rare large deviation events can be responsible for important failures of cellular machinery, leading to cancer [20] and the release of latent viruses from their quiescent state [21], [22]. The characterization of these events and the statistics of their occurrence is therefore of great importance, but also of great difficulty given standard simulation methods. The stochastic investigation of biological systems relies on the formulation of the chemical master equation (CME), a differential equation for the time evolution of probabilities [23], [24]. Despite the fairly straight-forward form and interpretation of the CME, it is not solvable exactly for any but systems that are "closed," in the sense that reactants are modeled to exist in a fixed volume and are not permitted to interact beyond the confines of that volume. However, models of cellular networks tend not to be closed, due to the prevalence of reactions that reintroduce into the reaction volume new copies of reactants through events such as new protein synthesis.

An alternative to solving the CME is to generate stochastic sample realizations of the process whose probability density function is described by the CME. This can be done, for instance, using standard Monte Carlo techniques, such as the Gillespie stochastic simulation algorithm (SSA) [25], [26]. The SSA has been used with great success in the study of a number of cellular networks. However, it has been repeatedly pointed out that the algorithm itself can become prohibitively inefficient when reactions occur so frequently that accounting for every event causes dramatic slowdown in simulation time. This effect is exacerbated by the presence of stiffness, i.e., the coexistence of drastically different time scales for the occurrence of the chemical reactions [27]. Furthermore, when investigating rare stochastic events, one needs to add to this inherent inefficiency the necessity of running extensive simulations in order to capture even one occurrence of the event of interest, a prohibitively expensive procedure. The same problem persists even when the above mentioned continuou-time discrete-state Markov description of cellular dynamics is replaced by a stochastic differential equation (SDE) description through diffusion approximations. The SDE is often referred to as a chemical Langevin equation (CLE). Indeed, the study of transitions between metastable steady states and rare events in stochastic systems is a difficult problem with a long history [28] and has many applications in biology [29]. Here again, we demonstrate how the algorithmic SOSTOOLS can be used to alleviate the computational burden involved in computing bounds on probabilities for the occurrence of rare biological events. As an illustration, we use a genetic switch implemented in the bacteriophage $\lambda$.

This paper is organized as follows. In Section II, we present a mathematical exposition of the algorithms and methods we use. We show how robust stability, model invalidation, and stochastic reachability analysis can be performed using the sum of squares technique in a unified manner. In Section III we illustrate the use of this general mathematical framework through two case studies. First, the robust stability of a HS model to parameter variations is assessed, followed by an invalidation of an erroneous model given data obtained by an adequate model. Then, we investigate the lytic/lysogenic cycles of bacteriophage lambda on $E$. coli using a stochastic model to answer stochastic reachability questions. Both examples are simple enough to be easily accessible but rich enough to be suggestive of the potential of the methods used. The paper is concluded in Section IV.

\section{MATHEMATICAL BACKGROUND}

In this section, we provide a basic description of the sum of squares formulation in the context of stability analysis, model validation/invalidation, and stochastic reachability problems.

\section{A. Robust Stability Analysis}

1) Stability and Lyapunov Functions: At the deterministic level, biological networks are usually modeled as a set of autonomous ordinary differential equations (ODEs) of the form

$$
\dot{x}=f(x)
$$

where $\dot{x}$ denotes the derivative of $x$ with respect to time, and $x=\left(x_{1}, \ldots, x_{n}\right)$ are the state variables. Here $f$ is a function that takes points in $\mathcal{D}$, a subset of $\mathbb{R}^{n}$ and maps them to $\mathbb{R}^{n}$, i.e., $f: \mathcal{D} \rightarrow \mathbb{R}^{n}$ with $\mathcal{D} \subseteq \mathbb{R}^{n}$. We assume further that $f$ is locally Lipschitz for $x \in \mathcal{D}$, a condition that guarantees local existence and uniqueness of solutions [30]. Let $x^{*}$ be an equilibrium point of (1), i.e., $f\left(x^{*}\right)=\dot{x}^{*}=0$. Without loss of generality, we assume that $x^{*}=0$ - a simple change of coordinates can achieve this — and study the stability properties of this equilibrium. We provide the following notions of stability.

Definition 1: Let $\|\cdot\|$ denote a norm in $\mathbb{R}^{n}$. The zero equilibrium of (1) is:

- stable, if for each $\epsilon>0$ there is $\delta=\delta(\epsilon)>0$ such that

$$
\|x(0)\|<\delta \Rightarrow\|x(t)\|<\epsilon, \quad \forall t \geq 0
$$

- asymptotically stable if it is stable, and $\delta$ can be chosen such that

$$
\|x(0)\|<\delta \Rightarrow \lim _{t \rightarrow \infty} x(t)=0 .
$$

These definitions of stability involve $\epsilon-\delta$ formulations; generally speaking, the stability condition requires that if the initial condition is close to the equilibrium, then the system trajectory will stay close to the equilibrium; the asymptotic 

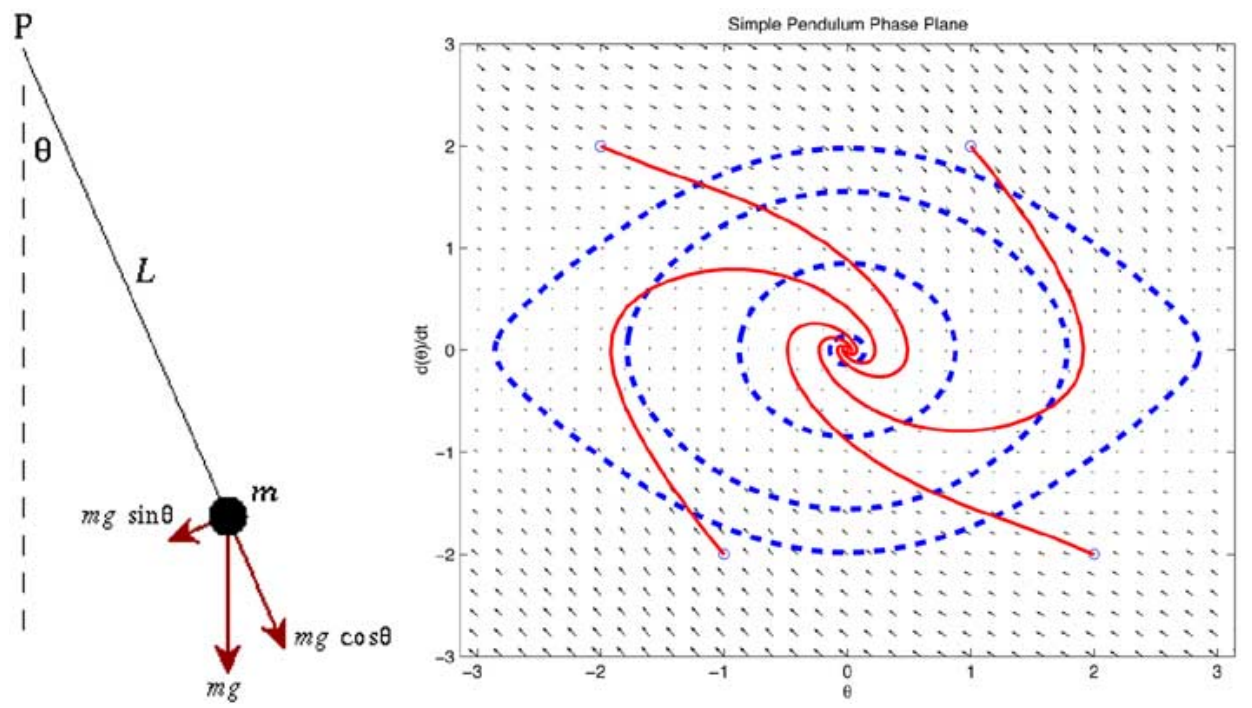

Fig. 1. (a) Simple pendulum with damping and (b) its phase plane. Arrows show the vector field directions, solid lines are sample trajectories with initial conditions indicated by "o." Dashed curves are level curves of the total energy of the system that serves as a Lyapunov function. The derivative condition on the Lyapunov function translates into the property that "arrows always cross inside the level curves of the Lyapunov function," which necessarily means that if a trajectory finds itself inside a level curve, it can never leave it.

stability condition not only asks for stability, but also asymptotic convergence to the equilibrium point. Such conditions give the impression that a complete description of the flow of the vector field is required to answer stability questions. It is fortunate that in many cases this is not essential; instead, stability can be proved directly by exhibiting a so-called Lyapunov function [30], [31].

Lyapunov functions are energy-like functions for a system. Consider a simple pendulum with damping, shown in Fig. 1. The total energy of the system is positive everywhere and is zero at the downward equilibrium. Moreover, as the system is left to evolve from any other initial condition, this energy function is nonincreasing which means that along the system evolution, energy is not added. By looking at how the total energy of such a system changes as the system evolves, we can conclude the stability of the equilibrium, as all initial conditions in a neighborhood of the equilibrium will have the same fate. Indeed, at the end of the 19th century, A. M. Lyapunov developed a stability theorem based on the above observation; through the properties of an appropriate energylike function that is widely referred to as a Lyapunov function) - being zero at the equilibrium, positive everywhere else, and its time derivative along the system's trajectories being nonincreasing - the stability of the equilibrium point follows.

The concept behind Lyapunov functions is central to the analysis of complex dynamical systems. Level curves of Lyapunov functions provide "trapping regions" that the trajectory can never leave-called regions of attraction of the equilibrium. Whereas simulation tries to follow the evolution of the system when released from a particular initial condition, the Lyapunov function constructs these nested regions which restrict the evolution of the system to within their boundaries-see Fig. 1. Under some technical conditions, the existence of this function has also been proven necessary for asymptotic stability [32]. More precisely, the conditions for this stronger notion of stability are stated in the following theorem.

Theorem 2 ([30]): Consider the system (1), and let $\mathcal{D} \subseteq$ $\mathbb{R}^{n}$ be a neighborhood of the origin. If there is a continuously differentiable function $V: \mathcal{D} \rightarrow \mathbb{R}$ such that the following two conditions are satisfied:

1) $V(x)>0$ for all $x \in \mathcal{D} \backslash\{0\}$ and $V(0)=0$, i.e., $V(x)$ is positive definite in $\mathcal{D}$;

2) $-\dot{V}(x)=-(\partial V / \partial x) f(x) \geq 0$ for all $x \in \mathcal{D}$, i.e., $\dot{V}(x)$ is negative semidefinite in $\mathcal{D}$;

then the origin is a stable equilibrium. If in condition (2) above, $\dot{V}(x)$ is negative definite in $\mathcal{D}$, then the origin is asymptotically stable. If $\mathcal{D}=\mathbb{R}^{n}$ and $V(x)$ is radially unbounded, i.e., $V(x) \rightarrow \infty$ as $\|x\| \rightarrow \infty$, then the result holds globally.

$V(x)$ in the previous theorem is called a Lyapunov function, while the surface $V(x)=a$ for some $a>0$ is called a Lyapunov surface. Therefore, the condition $\dot{V}(x) \leq 0$ indicates that when a trajectory of the system crosses $V(x)=a$, it subsequently stays within the set $\left\{x \in \mathbb{R}^{n} \mid V(x) \leq a\right\}$. Furthermore, the condition $\dot{V}(x)<0$ indicates that a trajectory moves from one Lyapunov surface to another with a smaller $a$, eventually approaching the origin.

In the case of linear time-invariant systems $\dot{x}=A x$ the stability properties can be characterized by the locations of the eigenvalues $\lambda_{i}$ of the matrix $A$, or equivalently, through a Lyapunov argument as follows.

Theorem 3 ([30]): The matrix $A$ is a stability matrix; that is $\operatorname{Re}\left(\lambda_{i}\right)<0$ for all eigenvalues $\lambda_{i}$ of $A$ if and only if for any given positive definite matrix $Q$ there exists a positive definite matrix $P$ that satisfies

$$
P A+A^{T} P=-Q .
$$

$P$ is unique and $V=x^{T} P x$ is a Lyapunov function for $\dot{x}=A x$. 
We see that the construction of the Lyapunov function in the case of linear systems is reduced to solving an appropriate algebraic Lyapunov equation (2). Alternatively, $P$ can be obtained by solving two linear matrix inequality (LMI) [33] conditions

$$
\begin{aligned}
P & >0, \\
A^{T} P+P A & <0 .
\end{aligned}
$$

A feasible $P$ exists if and only if $A$ is stable (all eigenvalues are in the open left half plane). LMIs are one possible formulation of semidefinite programs [34], which can be solved using algorithms with a worst case polynomial time complexity. This makes them particularly attractive for computation, and therefore constructing Lyapunov functions for linear systems is relatively simple.

In contrast to this, constructing Lyapunov functions for nonlinear systems is more difficult, as we will see in the sequel. This led to the development of other methods for assessing nonlinear system properties. For example, in Lyapunov's indirect method, one proceeds by linearizing the vector field about the equilibrium and the stability properties of the original nonlinear system are inferred from the stability properties of the linearized system. However, such stability results are valid only locally and the procedure is inconclusive when the linearized system has imaginary axis eigenvalues. Other methodologies involve absolute stability theory [35], linear parameter varying (LPV) embeddings [36]-[38], and integral quadratic constraint (IQC) formulations [39].

Next, we present a new computationally attractive methodology for nonlinear stability analysis that was first introduced by Parrilo in [40]. It is based on the sum of squares decomposition and uses semidefinite programming (LMIs) to construct functions that satisfy the conditions in Lyapunov's Theorem 2. The methodology generalizes the LMI method for constructing quadratic Lyapunov functions for linear time-invariant systems to constructing Lyapunov functions for nonlinear systems.

2) Algorithmic Construction of Lyapunov Functions Using Sum of Squares and SOSTOOLS: Here we concentrate on the construction of Lyapunov functions for nonlinear systems using Theorem 2. There are two main reasons for the absence of efficient algorithmic methodologies for constructing Lyapunov functions. First, the "terms" that should appear in a candidate Lyapunov function $V(x)$ are not known a priori. Second, testing the nonnegativity conditions in Theorem 2 is a difficult task even when a Lyapunov function is given, for example in the form of a polynomial. Sometimes, one can approach the first problem by resorting to intuition and prior knowledge of energy-like terms that are likely to appear in $V(x)$. However, the second problem is fundamentally intractable. It is closely related to the fact that testing nonnegativity of polynomials when their degree is greater than or equal to four is an $\mathcal{N} \mathcal{P}$-hard problem [41] and so algorithmic techniques to answer exactly the question "Given $p(x)$, is it nonnegative?" will most likely be impractical in the worst case. The related question "Given $p(x)$, is it a sum of squares?" can be answered using LMI techniques and is therefore algorithmically tractable. The sum of squares condition is sufficient for nonnegativity, and the approach that relaxes nonnegativity with sum of squares conditions was introduced by Parrilo [40] and has proven to be very powerful. We now present some background on sum of squares polynomials.

Definition 4: A multivariate polynomial $p(x), x \in \mathbb{R}^{n}$ is a sum of squares (SOS) if there exist polynomials $g_{i}(x)$, $i=1, \ldots, M$ such that $p(x)=\sum_{i=1}^{M} g_{i}^{2}(x)$.

An equivalent characterization of SOS polynomials is given in the following proposition, the proof of which can be found in [40].

Proposition 5 [40]: A polynomial $p(x)$ of degree $2 d$ is an SOS if and only if there exists a positive semidefinite matrix $Q$ and a vector $Z(x)$ containing monomials in $x$ of degree $\leq d$ so that

$$
p(x)=Z(x)^{T} Q Z(x) .
$$

This proposition states that instead of searching for the $g_{i}(x)$ that make up $p(x)$ in the definition above, one can equivalently search for a matrix $Q$ that is positive semidefinite so that $p(x)=Z(x)^{T} Q Z(x)$. If such a $Q$ is found, then the polynomial is an SOS, and if it is not found, then the polynomial is not an SOS. The vector $Z(x)$ can be constructed by looking at the monomials that appear in $p(x)$ and its degree.

Since $p(x)$ being an SOS is equivalent to $Q \geq 0$ subject to some affine conditions between the coefficients of the monomials in $p(x)$ and the elements of the unknown matrix $Q$, the problem of finding a $Q$ which proves that $p(x)$ is an SOS is indeed an LMI [40]. Note that if a polynomial $p(x)$ is a sum of squares, then it is globally nonnegative. The converse is not always true: not all positive semidefinite polynomials can be written as SOS-in fact, as mentioned earlier, testing global nonnegativity of a polynomial $p(x)$ is known to be $\mathcal{N P}$-hard when the degree of $p(x)$ is greater than 4 [41], whereas checking whether $p$ can be written as an SOS is computationally tractable - it can be formulated as an LMI which has a worst case polynomial-time complexity. Nevertheless, the gap between nonnegativity and SOS appears small in practical problems [42]. The construction of the LMI related to the SOS conditions can be performed efficiently using SOSTOOLS [43], a software that formulates general sum of squares programs as semidefinite programs (SDPs) and calls semidefinite programming solvers to solve them. If the monomials in the polynomial $p(x)$ have unknown coefficients then the search for feasible values of those coefficients such that $p(x)$ is nonnegative is also an SDP, a fact that is important for the construction of Lyapunov functions.

Let us now return to the problem of constructing Lyapunov functions for nonlinear systems, and assume for simplicity that $f(x)$ is a polynomial vector field, i.e., $f_{i}(x)$ are polynomials in $x=\left(x_{1}, \ldots, x_{n}\right)$ for $i=1, \ldots, n$. Suppose that we also wish to construct a $V(x)$ that is also polynomial in $x$. In this case, the two conditions in Theorem 2 become polynomial nonnegativity conditions. To circumvent the difficult 
task of testing them directly, we can restrict our attention to the case in which the two conditions admit SOS decompositions.

In order to impose positive definiteness (rather than positive semidefiniteness) on $V(x)$, we construct an auxiliary positive definite "shaping" function $\varphi(x)$ as follows:

$$
\varphi(x)=\sum_{i=1}^{n} \sum_{j=1}^{d} \epsilon_{i j} x_{i}^{2 j}, \quad \sum_{j=1}^{m} \epsilon_{i j} \geq \gamma, \forall i=1, \ldots, n
$$

where $\gamma>0$, and $\epsilon_{i j} \geq 0 \forall i, j$. This makes $\varphi(x)>0$, i.e., positive definite. If we then impose $V(x)-\varphi(x)$ to be an SOS, we get the obvious relation

$$
V(x)-\varphi(x) \geq 0 \Rightarrow V(x) \geq \varphi(x)>0 .
$$

Overall, we have the following proposition.

Proposition 6: Given a polynomial $V(x)$ of degree $2 d$, let $\varphi(x)$ be given by (3). Then, the condition

$$
V(x)-\varphi(x) \text { is a sum of squares }
$$

guarantees the positive definiteness of $V(x)$.

In light of this proposition, testing global stability of $\mathcal{D}=$ $\mathbb{R}^{n}$ following the conditions of Theorem 2 can be formulated directly as SOS conditions. It can be specifically formulated as the following sum of squares program.

Program 7: To construct a Lyapunov function for system

Find a polynomial $V(x), \quad V(0)=0$

and a positive definite function $\varphi(x)$ of the form (3)

such that

$$
\begin{aligned}
& V(x)-\varphi(x) \text { is SOS } \\
& -\frac{\partial V}{\partial x} f(x) \text { is SOS. }
\end{aligned}
$$

$V(x)$ found this way is a Lyapunov function for system (1) and the zero equilibrium of (1) is stable, since the above program guarantees that $V(x)$ is positive definite and also that $\dot{V}(x)$ is negative semidefinite. Note also that by construction $\varphi(x)$ is radially unbounded; therefore, $V(x)$ will also be radially unbounded, and the stability property holds globally [30]. Also, if condition (7) is replaced by

$$
-\frac{\partial V}{\partial x} f(x)-\psi(x) \text { is SOS }
$$

where $\psi(x)$ is a positive definite polynomial constructed as per (3), then $\dot{V}(x)$ is negative definite and the origin is globally asymptotically stable.

3) Stability of Constrained Systems-Robust Stability Analysis: To tackle more biologically relevant problems, we extend here Lyapunov's theorem to systems that evolve under equality, inequality, and integral constraints. This is a very general class of systems, special cases of which are differential algebraic equations, robust stability analysis, and performance evaluation. Furthermore, this extension allows for the treatment of nonpolynomial vector fields.

Inequality constraints arise naturally in biological networks, as the states in biochemical reactions (concentrations of species) are always nonnegative. The same type of inequality constraints can be further used to describe uncertain parameter sets for the study of robust stability of systems in the presence of parametric uncertainty. Equality constraints also prove useful in robust stability analysis where they appear as constraints guaranteeing that the equilibrium of the system is at the origin. Equality constraints can also naturally appear in systems that are constrained to evolve over a manifold [44]. For example, equality constraints in biological systems can arise due to conservation laws-usually in the form of mass balance equations. Finally, integral type constraints can also be incorporated. In particular, one can consider integral quadratic constraints (IQCs) [39], which provide a rich framework that encapsulates many types of uncertainty and unmodeled dynamics: dynamic, time-varying and $L_{2}$ bounded uncertainty, just to name a few. Performance calculations such as $L_{2}$ input-output gain estimation can also be formulated using IQCs.

To put these ideas in a more rigorous setting, we consider the nonlinear system

$$
\dot{x}=f(x, u)
$$

with the following inequality, equality, and integral constraints that are satisfied by $x$ and $u$

$$
\begin{aligned}
a_{i_{1}}(x, u) & \leq 0, \text { for } i_{1}=1, \ldots, N_{1}, \\
b_{i_{2}}(x, u) & =0, \text { for } i_{2}=1, \ldots, N_{2}, \\
\int_{0}^{T} c_{i_{3}}(x, u) d t & \leq 0, \text { for } i_{3}=1, \ldots, N_{3}, \text { and } \forall T \geq 0 .
\end{aligned}
$$

Here $x \in \mathbb{R}^{n}$ is the state of the system, and $u \in \mathbb{R}^{m}$ is a collection of auxiliary variables (such as inputs, nonpolynomial functions of states, uncertain parameters, etc). We assume that $f(x, u)$, apart from the required Lipschitz conditions for existence of solutions, has no singularity in $\mathcal{D}$, where $\mathcal{D} \subseteq \mathbb{R}^{n+m}$ is defined as

$\mathcal{D}=\left\{(x, u) \in \mathbb{R}^{n+m} \mid a_{i_{1}}(x, u) \leq 0, b_{i_{2}}(x, u)=0, \forall i_{1}\right.$ and $\left.i_{2}\right\}$ 
Without loss of generality, it is also assumed that $f(x, u)=0$ for $x=0$ and $u \in \mathcal{D}_{u}^{0}$, where

$$
\mathcal{D}_{u}^{0}=\left\{u \mathbb{R}^{m} \mid(0, u) \in \mathcal{D}\right\} .
$$

The following theorem is an extension of Lyapunov's stability theorem, and can be used to prove that the origin is a stable equilibrium of the above system. It uses a technique reminiscent of the well-known S-procedure [45] in nonlinear and robust control theory [33], which is similar to adjoining the equality, inequality and IQC conditions to the Lyapunov conditions using appropriate multipliers.

Theorem 8 [13]: Suppose that for system (9), there exist functions $V(x), p_{1_{i_{1}}}(x, u) \geq 0, p_{2_{i_{1}}}(x, u) \geq 0, q_{1_{i_{2}}}(x, u)$, $q_{2_{i_{2}}}(x, u)$, and constants $r_{i_{3}} \geq 0$ such that

$$
\begin{aligned}
& V(x)+\sum p_{1_{i_{1}}}(x, u) a_{i_{1}}(x, u) \\
& \quad+\sum q_{1_{i_{2}}}(x, u) b_{i_{2}}(x, u)>0 \\
& -\frac{\partial V}{\partial x} f(x, u)+\sum p_{2_{i_{1}}}(x, u) a_{i_{1}}(x, u) \\
& \quad+\sum q_{2_{i_{2}}}(x, u) b_{i_{2}}(x, u)+\sum r_{i_{3}} c_{i_{3}}(x, u) \geq 0
\end{aligned}
$$

Then the origin of the state space is a stable equilibrium of the system.

Proof: If condition (13) is fulfilled, then we have that in $\mathcal{D}$,

$V(x)>-\sum p_{1_{i_{1}}}(x, u) a_{i_{1}}(x, u)-\sum q_{1_{i_{2}}}(x, u) b_{i_{2}}(x, u) \geq 0$.

Therefore, $V(x)>0$ in $\mathcal{D}$, where $a_{i_{1}}(x, u)$ and $b_{i_{2}}(x, u)$ satisfy (10), (11).

Condition (14) can be integrated from time $t=0$ to $t=T$ to obtain

$$
\begin{aligned}
V(x(0))-V(x(T)) & \geq-\sum \int_{0}^{T}\left\{p_{2_{i_{1}}}(x, u) a_{i_{1}}(x, u)\right. \\
& \left.+r_{i_{3}} c_{i_{3}}(x, u)\right\} d t
\end{aligned}
$$

where we have used the fact that $a_{i_{1}}(x, u), b_{i_{2}}(x, u)$, and $c_{i_{3}}(x, u)$ satisfy (10)-(12).

This shows that the Lyapunov function is nonincreasing along the trajectories of the system and is positive definite in $\mathcal{D}$. Therefore, the conditions for Lyapunov stability (see Theorem 2) are satisfied. The rest of the proof is similar to the proof of Lyapunov's theorem, which can be found in many standard textbooks, e.g., [30]

Concisely put, in order to test that the Lyapunov conditions hold when the constraints (10)-(12) are satisfied, we adjoined these constraints using appropriate multipliers to the Lyapunov conditions of Theorem 2.
When the vector field $f(x, u)$ is rational, i.e., $f(x, u)=$ $n(x, u) / d(x, u)$ with $d(x, u) \neq 0$ in $\mathcal{D}$, condition (14) can be multiplied by the nonvanishing denominator. Rational vector fields are common in biological systems, usually arising from Michaelis-Menten approximations.

Theorem 8 can now be used to analyze various cases of systems with constraints. Now, we demonstrate how $V(x)$ in Theorem 8 can be constructed using the SOS technique. For this, we need to make some assumptions.

- The vector field $f_{x}(x, u)$ is assumed to be polynomial or rational, and the constraint functions $a_{i_{1}}(x, u)$, $b_{i_{2}}(x, u), c_{i_{3}}(x, u)$ are assumed to be polynomial. This assumption may be removed through a recasting process [46].

- We search for a bounded degree polynomial Lyapunov function $V$ and multipliers $p_{i_{1}}, q_{i_{1}}, i_{1}=1, \ldots, N_{1}$ and $p_{i_{2}}, q_{i_{2}}, i_{2}=1, \ldots, N_{2}$.

Under these assumptions, the search for a Lyapunov function can be relaxed by the following proposition in which all " $\geq$ " conditions are essentially relaxed to SOS conditions, making their search computationally efficient using semidefinite programming and SOSTOOLS.

Proposition 9: Suppose that for system (9) with $f(x, u)=$ $n(x, u) / d(x, u)$ where $n(x, u)$ and $d(x, u)$ are polynomials and $d(x, u)>0$ in $\mathcal{D}$, there exist polynomial functions $V(x)$, $p_{1_{i_{1}}}(x, u), p_{2_{i_{1}}}(x, u), q_{1_{i_{2}}}(x, u), q_{2_{i_{2}}}(x, u)$, a positive definite function $\varphi(x)$ of the form given in (3) and constants $r_{i_{3}} \geq 0$ such that

$$
\begin{aligned}
& V(x)+\sum p_{1_{i_{1}}}(x, u) a_{i_{1}}(x, u) \\
& \quad+\sum q_{1_{i_{2}}}(x, u) b_{i_{2}}(x, u)-\varphi(x) \text { is SOS } \\
& p_{1_{i_{1}}}(x, u) \text { is SOS for } i_{1}=1, \ldots, N_{1}, \\
& p_{2_{i_{1}}}(x, u) \text { is SOS for } i_{1}=1, \ldots, N_{1}, \\
& d(x, u)\left(-\frac{\partial V}{\partial x} f(x, u)+\sum p_{2_{i_{1}}}(x, u) a_{i_{1}}(x, u)\right. \\
& \left.\quad+\sum q_{2_{i_{2}}}(x, u) b_{i_{2}}(x, u)+\sum r_{i_{3}} c_{i_{3}}(x, u)\right)
\end{aligned}
$$

is SOS.

Then the origin of the state space is a stable equilibrium of the system.

It is now clear how $V(x), p_{1_{i_{1}}}(x, u), p_{2_{i_{1}}}(x, u)$, $q_{1_{i_{2}}}(x, u), q_{2_{i_{2}}}(x, u)$, the constants $r_{i_{3}}$ and the positive definite function $\varphi(x)$ can be constructed using SOSTOOLS [43], and a program similar to Program 7 can be constructed.

\section{B. Model Invalidation}

Model validation provides a way to evaluate the ability of a proposed model to represent observed system behaviors. However, as often pointed out in the literature, "model validation" is actually a misnomer [47]-[49]. It is impossible to validate a model, because to do so requires an infinite number of experiments and data. The role of model validation techniques is rather to invalidate a model, by proving that some 
experimental data are inconsistent with the model, thus indicating that a refinement of the model is required. Beside modeling, model validation is related to and has many applications in the areas of system identification, fault detection, prediction, and verification.

In this section, we present a methodology recently developed for invalidation of continuous-time nonlinear models with uncertain parameters [7]. The methodology is based on functions of state-parameter-time termed barrier certificates. The existence of a barrier certificate generates a contradiction between model and some time-domain experimental data, in the sense that some level sets of this certificate act as barriers between possible model trajectories and data. With this methodology, model validation of a very large class of continuous-time models, including differential-algebraic models [50], models with uncertain inputs [39], models with memoryless and dynamic uncertainties [30], [39], hybrid models [51], and their combinations. Moreover, similar to Lyapunov functions, barrier certificates can be computed using the sum of squares decomposition and semidefinite programming, e.g., using the SOSTOOLS software [43].

In the simplest setting, consider again the system of ordinary differential equations

$$
\dot{x}(t)=f(x(t), p, t)
$$

where $x(t) \in \mathbb{R}^{n}$ is the vector of state variables, $t$ is time, and $p \in \mathbb{R}^{m}$ is the parameter vector, assumed to take its value in a set $P \subset \mathbb{R}^{m}$. Let an experiment be performed with the real system, and two measurements be taken at time $t=0$ and $t=T$. Suppose that these measurements indicate that $x(0) \in X_{0}$ and $x(T) \in X_{T}$, where both $X_{0}$ and $X_{T}$ are subsets of $\mathbb{R}^{n}$. In addition, assume that $x(t) \in X$ for all $t \in[0, T]$, where $X \subseteq \mathbb{R}^{n}$. The invalidation problem can then be stated as follows.

Problem 10: Given the model (19), parameter set $P$, and trajectory information $\left\{X_{0}, X_{T}, X\right\}$, prove that for all possible parameter $p \in P$, the model (19) cannot produce a trajectory $x(t)$ such that $x(0) \in X_{0}, x(T) \in X_{T}$, and $x(t) \in X, \forall t \in[0, T]$.

If such a proof in Problem 10 can be found, then we say that the model (19) and parameter set $P$ are invalidated by $\left\{X_{0}, X_{T}, X\right\}$.

Traditional approaches for solving this problem include exhaustive simulation of (19) using parameters $p$ and initial conditions $x(0)$ sampled randomly from $P$ and $X_{0}$. If after many such simulations no trajectory $x(t)$ that satisfies the initial hypothesis can be found, then an inconsistency is concluded. Indeed simulation (possibly after parameter fitting) is a good way for proving that a model can reproduce some behaviors of the system it represents. However, for proving inconsistency, the required number of simulation runs will soon become prohibitive. Moreover, a proof by simulation alone is never exact, simply because it is impossible to test all $p$ and $x(0)$.

Instead of exhaustive simulations, our method relies on the existence of a function of state-parameter-time, which we term barrier certificate. A barrier certificate gives an exact proof of inconsistency by providing a barrier between possible trajectories of the model starting at $X_{0}$ and the final measurement $X_{T}$. This is accomplished without performing any simulation nor computing the flow of the model. The method is summarized in the following theorem.

Theorem 11 ([7]): Let the model (19) and the sets $P, X_{0}$, $X_{T}, X$ be given, with $f(x, p, t)$ being continuous in $x$ and $t$. Suppose that there exists a real-valued function $B(x, p, t)$ that is differentiable with respect to $x$ and $t$, such that

$$
\begin{aligned}
& B\left(x_{T}, p, T\right)-B\left(x_{0}, p, 0\right)>0 \\
& \forall\left(x_{T}, x_{0}, p\right) \in X_{T} \times X_{0} \times P, \\
& \frac{\partial B}{\partial x}(x, p, t) f(x, p, t)+\frac{\partial B}{\partial t}(x, p, t) \leq 0 \\
& \forall(x, p, t) \in X \times P \times[0, T] .
\end{aligned}
$$

Then the model (19) and its associated parameter set $P$ are invalidated by $\left\{X_{0}, X_{T}, T\right\}$. (In the sequel, we will call the function $B(x, p, t)$ a barrier certificate.

The proof of the theorem follows from a simple contradiction. In particular, according to the experimental data and (20), we have the value of $B(x(t), p, t)$ at time $t=T$ greater than its value at time $t=0$. On the other hand, (21) implies that this value should not increase along time when $x(t)$ is a trajectory of the model (19). Thus, we can conclude that the model is inconsistent with the experimental data. The above approach can be easily extended to handle more than two measurements. In addition, as mentioned at the beginning of the section, this approach can be used to handle a larger class of models. See [7] for details.

Similar to the case of Lyapunov functions, construction of barrier certificates is in general not easy. However, for models with polynomial vector fields and sets $P, X_{T_{i}}, X$, $U_{i}$ described by polynomial equalities and inequalities, a tractable computational relaxation for constructing barrier certificates exists. The relaxation is provided by the sum of squares decomposition, much in the spirit of the algorithmic construction of Lyapunov functions for nonlinear systems presented in Section II-A2.

More concretely, consider the model (19) with a polynomial $f(x, p, t)$ and parameter set $P$ defined as follows:

$$
P=\left\{p \in \mathbb{R}^{m}: g_{P, i}(p) \geq 0 \quad \forall i \in I_{P}\right\}
$$

where the $g_{P, i}(p)$ 's are polynomials in $p$, and $I_{P}$ is an index set. For example, when the elements of $p$ take their values on the intervals $\underline{p_{i}} \leq p_{i} \leq \overline{p_{i}}$, we may define

$$
g_{P, i}(p)=\left(p_{i}-\underline{p_{i}}\right)\left(\overline{p_{i}}-p_{i}\right), \quad \text { for } i \in I_{P}=\{1, \ldots, m\}
$$

Similarly, let the trajectory data be defined by

$$
X_{0}=\left\{x_{0} \in \mathbb{R}^{n}: g_{0, i}\left(x_{0}\right) \geq 0 \quad \forall i \in I_{0}\right\}
$$




$$
\begin{aligned}
X_{T} & =\left\{x_{T} \in \mathbb{R}^{n}: g_{T, i}\left(x_{T}\right) \geq 0 \quad \forall i \in I_{T}\right\}, \\
X & =\left\{x \in \mathbb{R}^{n}: g_{X, i}(x) \geq 0 \quad \forall i \in I_{X}\right\} .
\end{aligned}
$$

Then a barrier certificate can be computed by solving the convex optimization problem given in the following program.

Program 12: Let the polynomial vector field $f(x, p, t)$ and the sets $P, X_{0}, X_{T}, X$ in (22)-(25) be given. To invalidate the model (19), find a polynomial $B(x, p, t)$, a positive number $\epsilon$, and sums of squares $M_{P, i}\left(x_{0}, x_{T}, p\right)$, $M_{0, i}\left(x_{0}, x_{T}, p\right), \quad M_{T, i}\left(x_{0}, x_{T}, p\right), \quad N_{P, i}(x, p, t)$, $N_{X, i}(x, p, t), N_{t}(x, p, t)$, such that the expressions

$$
\begin{aligned}
& B\left(x_{T}, p, T\right)-B\left(x_{0}, p, 0\right)-\epsilon-\sum_{i \in I_{P}} M_{P, i}\left(x_{0}, x_{T}, p\right) g_{P, i}(p) \\
& \quad-\sum_{i \in I_{0}} M_{0, i}\left(x_{0}, x_{T}, p\right) g_{0, i}\left(x_{0}\right) \\
& \quad-\sum_{i \in I_{T}} M_{T, i}\left(x_{0}, x_{T}, p\right) g_{T, i}\left(x_{T}\right)
\end{aligned}
$$

and

$$
\begin{gathered}
-\frac{\partial B}{\partial x}(x, p, t) f(x, p, t)-\frac{\partial B}{\partial t}(x, p, t)-\sum_{i \in I_{P}} N_{P, i}(x, p, t) g_{P, i}(p) \\
-\sum_{i \in I_{X}} N_{X, i}(x, p, t) g_{X, i}(x)-N_{t}(x, p, t)\left(T t-t^{2}\right)
\end{gathered}
$$

are sums of squares. (In this case, $B(x, p, t)$ satisfies the conditions (20), (21) of Theorem 11, and therefore $B(x, p, t)$ is a barrier certificate.)

At this point, we would like to remark that although this computational approach assumes that the description of the model, parameter set, and data sets are polynomial, nonpolynomial descriptions can be handled (although possibly with some conservatism), and nonpolynomial barrier certificates can be constructed by recasting of variables as proposed in [46].

\section{Stochastic Verification}

Computing bounds on probability in inherently stochastic biological systems, as well as the statistics for the occurrence or lack thereof of decisive or catastrophic events is a crucial problem. However, such computations are usually very challenging, and can be carried analytically or numerically only for a handful of examples. In this section, a technique based on the sum of squares approach that was recently developed [52], [53] for the algorithmic computation of probability bounds on the occurrence of these events will be described.

For our exposition, we consider a complete probability space $(\Omega, \mathcal{F}, P)$ and a standard $\mathbb{R}^{m}$-valued Wiener process $w$ defined on this space [54]. The class of models that we use is stochastic differential equations of the form

$$
d x(t)=f(x(t)) d t+g(x(t)) d w(t)
$$

where $x(t) \in \mathbb{R}^{n}$, and $t \geq 0$. We denote the state space, the set of initial states, and the set of decisive/unsafe states by $\mathcal{X}$, $\mathcal{X}_{0}$, and $\mathcal{X}_{u}$, respectively. All of these are subsets of $\mathbb{R}^{n}$ and assumed compact. To guarantee the existence and uniqueness of solution, we will also assume that both $f(x)$ and $g(x)$ satisfy the local Lipschitz condition and the linear growth condition on $\mathcal{X}$. For bounded $\mathcal{X}$, the last condition can be replaced by the boundedness of $f$ and $g$ on $\mathcal{X}$. It can then be shown that the process $x(t)$ described above is a strong Markov process.

Since in general the process $x(t)$ is not guaranteed to always lie inside the set $\mathcal{X}$, we define the stopped process $\tilde{x}(t)$ corresponding to $x(t)$ and $\mathcal{X}$ as follows.

Definition 13: Suppose that $\tau$ is the first time of exit of $x(t)$ from the open set $\operatorname{Int}(\mathcal{X})$. The stopped process $\tilde{x}(t)$ is defined by

$$
\tilde{x}(t)= \begin{cases}x(t) & \text { for } t<\tau \\ x(\tau) & \text { for } t \geq \tau\end{cases}
$$

The stopped process $\tilde{x}(t)$ satisfies various properties. For example, it inherits the right continuity and strong Markovian property of $x(t)$. Furthermore, in most cases the so-called infinitesimal generator corresponding to $\tilde{x}(t)$ is identical to the one corresponding to $x(t)$ on the $\operatorname{set} \operatorname{Int}(\mathcal{X})$, and is equal to zero outside of this set [55]. This will be implicitly assumed in our analysis.

Having defined the system and the stopped process $\tilde{x}(t)$, we can now state the stochastic verification problem as follows.

Problem 14: Given the system (28) and the sets $\mathcal{X}, \mathcal{X}_{0}$ and $\mathcal{X}_{u}$, compute an upper bound for the probability of a process $\tilde{x}(t)$ starting at $\mathcal{X}_{0}$ to reach $\mathcal{X}_{u}$. In other words, find $\gamma \in[0,1]$ such that $P\left\{\tilde{x}(t) \in \mathcal{X}_{u}\right.$ for some $\left.t \geq 0 \mid \tilde{x}(0)=x_{0}\right\} \leq \gamma$ for all $x_{0} \in \mathcal{X}_{0}$.

Obviously, it is of interest to obtain an upper bound $\gamma$ that is as tight as possible. Our approach to solve the above problem is based on finding an appropriate function $B(x)$ from which we can deduce an upper bound $\gamma$. We will also call the function $B(x)$ a barrier certificate, as it will need to satisfy some conditions that can be considered as the stochastic counterpart of the conditions in Section II-B. For example, instead of requiring the value of $B(x(t))$ to be nonincreasing along time, we ask that the expected value of $B(x(t))$ decreases or stays constant as time increases. A process satisfying such a property is called a supermartingale (see [54] for the technical definition). Using a known supermartingale inequality [55], the probability bound can then be inferred from $B(x)$, as summarized in the following theorem.

Theorem 15 ([53]): Let the stochastic differential equation (28) and the sets $\mathcal{X}, \mathcal{X}_{0}, \mathcal{X}_{u}$ be given, and consider the stopped process $\tilde{x}(t)$. Suppose that there exists a twice continuously differentiable function $B: \mathbb{R}^{n} \rightarrow \mathbb{R}$, such that

$$
B(x) \geq 0 \forall x \in \chi,
$$




$$
\begin{aligned}
& B(x) \geq 1 \forall x \in \chi_{u}, \\
& B(x) \leq \gamma \forall x \in \chi_{0}, \\
& \frac{\partial B}{\partial x}(x) f(x)+\frac{1}{2} \text { Trace }\left(g^{T}(x) \frac{\partial^{2} B}{\partial x^{2}}(x) g(x)\right) \\
& \quad \leq 0 \forall x \in \chi
\end{aligned}
$$

then

$$
P\left\{\tilde{x}(t) \in \chi_{u} \text { for some } t \geq 0 \mid \tilde{x}(0)=x_{0}\right\} \leq \gamma
$$

for all $x_{0} \in \mathcal{X}_{0}$.

It should be noted that the value of $\gamma$ will be at most equal to one, as when $\gamma=1$, the function $B(x)=1$ will satisfy the above conditions. The proof of Theorem 15 can be found in [53], but the intuitive idea is as follows. Condition (32) implies that the process $B(\tilde{x}(t))$ is a supermartingale, and therefore its value is likely to stay constant or decrease as time increases. When the system starts from a lower initial value of $B(x)$ (i.e., as $\gamma$ gets smaller) it becomes less likely for the trajectory to reach the decisive/unsafe states, on which the value of $B(x)$ is greater than or equal to one. This is quantified by the probability bound (33).

With regard to computation, an upper bound $\gamma$ and a barrier certificate $B(x)$ which certifies the upper bound can be computed by formulating conditions (29)-(32) as a sum of squares optimization problem, in the same spirit as Program 12. Furthermore, $\gamma$ can be chosen as the objective function whose value is to be minimized. The minimum value of $\gamma$ obtained from the optimization will be the tightest upper bound for a given set of candidate barrier certificates. Obviously, as we include more candidate barrier certificates to this set, we may get a better bound, although there is a tradeoff between using a larger set and the computational complexity of finding a barrier certificate within the set.

Other classes of stochastic systems, e.g., switching diffusion processes, can be handled in a similar fashion, by using the suitable generator for the class and modifying the conditions for $B(x)$ appropriately. See [53].

\section{ApPlicAtion to Gene RegUlatory Networks}

In order to put the biological models of this section in context, we present a brief introduction to the cellular processes involved in gene expression, in addition to the concept of a gene regulatory network.

\section{A. Gene Regulatory Networks and the Central Dogma of Molecular Biology}

The synthesis of cellular proteins is a multistep process that involves the use of various cellular machines [56]. One very important machine in bacteria is the so-called RNA polymerase (RNAP). RNAP is an enzyme that can be recruited to transcribe any given gene. However, RNAP bound to regulatory sigma factors recognizes specific sequences in the DNA, referred to as the promoter. Whereas the role of RNAP is to transcribe genes, the main role of $\sigma$ factors is to recognize the promoter sequence and signal to RNAP in order to initiate the transcription of the appropriate genes. The transcription process itself consists of synthesizing a messenger RNA (mRNA) molecule that carries the information encoded by the gene. Here, RNAP acts as a "reading head" transcribing DNA sequences into mRNA. Once a few nucleotides on the DNA have been transcribed, the $\sigma$-factor molecule dissociates from RNAP, while RNAP continues transcribing the genes until it recognizes a particular sequence called a terminator sequence. At this point, the mRNA is complete and RNAP disengages from the DNA. During the transcription process, ribosomes bind to the nascent mRNA and initiate translation of the message. The process of translation consists of sequentially assembling amino acids in an order that corresponds to the mRNA sequence, with each set of three nucleotides corresponding to a single unique amino acid. This combined process of gene transcription and mRNA translation constitutes gene expression, and is often referred to as the central dogma of molecular biology.

Gene regulatory networks can be broadly defined as groups of genes that are activated or deactivated by particular signals and stimuli, and as such produce or halt the production of certain proteins. Through combinatorial logic at the gene or the end-product protein level, these networks orchestrate their operation to regulate certain biological functions such as metabolism, development, or the cellular clocks. Regulation schemes in gene regulatory networks often involve positive and negative feedback loops. A simple scheme consists, for example, of a protein that binds to the promoter of its own gene and shields it from the RNAP- $\sigma$ complex, thereby autoregulating its own production. When interfaced and connected together according to a certain logic, a network of such building blocks (and possibly others possessing different architectures and components) generates intricate systems that possess a wide range of dynamical behaviors and functionalities.

\section{B. Robust Stability Analysis of the Heat Shock Response in E. coli}

In this section, we describe the biology of the heat shock response (HS) system, an essential cellular mechanism conserved in most organisms. Extensive experimental probing of the architecture of the HS system has unraveled a number of feedback and feedforward loops whose presence posed many essential questions. First, and perhaps foremost, does the current knowledge span all the structural components and interactions in the system or did experimental investigation miss crucial parts? If so, can a model describing the known components identify this knowledge gap when contrasted with data? Even if such investigations confirm the completeness of our knowledge of the system, can we decipher the salient features of its architecture and identify the functional roles of its parts. For example, what is the functional relevance of the seemingly redundant loops in the HS system? Is the system robust to operational fluctuations? If so, how is this robustness connected to the architecture? 


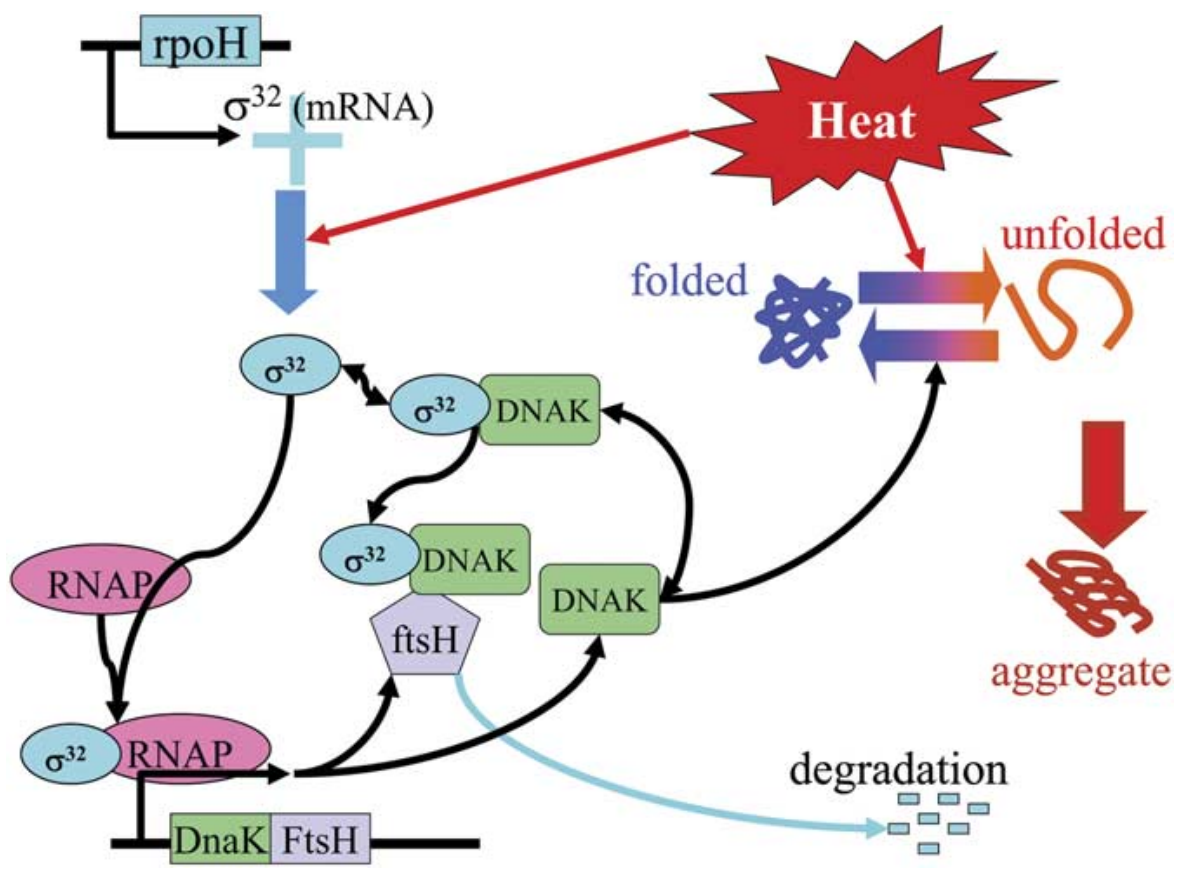

Fig. 2. Molecular implementation of the HS response system.

These questions are of course of general relevance to many biological systems, but are at the same time immensely challenging. Here, we use the HS response as a case study to formulate and address concisely particular aspects of robustness analysis and model invalidation, all in the context of our new mathematical machinery. The HS response is particularly suited for such an investigation because of the wealth of experimental and mathematical information available for the system [11].

1) The Heat Shock Response in E. coli: High temperatures cause cell proteins to unfold from their normal shapes, resulting in malfunctioning and eventually death of the cell. Cells have evolved gene regulatory mechanisms to counter the effects of HS by expressing specific genes that encode HS proteins (hsps) whose role is to help the cell survive the consequence of the shock. In E. coli, the HS response is implemented through an intricate architecture of feedback loops centered on the $\sigma$-factor that regulates the transcription of the HS proteins under normal and stress conditions. The enzyme RNA polymerase (RNAP) bound to this regulatory sigma factor, $\sigma^{32}$, recognizes the HS gene promoters and transcribes specific HS genes. The HS genes encode predominantly molecular chaperones (DnaK, DnaJ, GroEL, GrpE, etc.) that are involved in refolding denatured proteins and proteases (Lon, FtsH, etc.) that function to degrade unfolded proteins. At physiological temperatures (30 ${ }^{\circ} \mathrm{C}-37{ }^{\circ} \mathrm{C}$ ), there is very little $\sigma^{32}$ present and hence little transcription of the HS genes. When bacteria are exposed to high temperatures, $\sigma^{32}$ first rapidly accumulates, allowing increased transcription of the HS genes and then declines to a new steady state level characteristic of the new growth temperature. There are two mechanisms by which $\sigma^{32}$ levels are increased when the temperature is raised [57]. First, the translation rate of the rpoH mRNA (encoding $\sigma^{32}$ ) increases immediately, resulting in a fast tenfold increase in the concentration of $\sigma^{32}$ [58]. This mechanism implements what we refer to as the feedforward control loop. Second, during steady state growth, $\sigma^{32}$ is rapidly degraded $\left(t_{1 / 2}=1 \mathrm{~min}\right)$, but is stabilized for the first 5 min after temperature upshift, so that its concentration rapidly increases. In vivo evidence is consistent with the following titration model for the HS response. DnaK and its cochaperone DnaJ are required for the rapid degradation of $\sigma^{32}$ by the HS protease FtsH. Raising the temperature produces an increase in the cellular levels of unfolded proteins that then titrate DnaK/J away from $\sigma^{32}$, allowing it to bind to RNA polymerase (resulting in increased trancription) and stabilizing it in the process. Together, increased translation and stabilization lead to a transient 15-20-fold increase in the amount of $\sigma^{32}$ at the peak of the HS response. The accumulation of high levels of HS proteins leads to the efficient refolding of the denatured proteins thereby decreasing the pool of unfolded protein, freeing up DnaK/J to sequester this protein from RNA polymerase. This implements what is referred to as a sequestration feedback loop. Furthermore, this sequestration itself promotes the degradation of $\sigma^{32}$ and results in feedback regulated degradation, mainly by the protease FtsH. We refer to this as the FtsH degradation feedback loop. The overall result is a decrease in the concentration of $\sigma^{32}$ to a new steady state concentration that is dictated by the balance between the temperature-dependent translation of the rpoH mRNA and the level of $\sigma^{32}$ activity modulated by the hsp chaperones and proteases acting in a negative feedback fashion. The molecular interactions describing the HS response are pictorially illustrated in Fig. 2.

2) A Reduced Order Model for the HS Response: In a previous study, we have developed a detailed deterministic mathematical model for the heat stress response in E. coli 
Table 1

Parameter Values for Heat Shock Model

\begin{tabular}{|l|l|}
\hline Parameter & Value \\
\hline \hline$K_{d}$ & 3 min $^{-1}$ \\
$\alpha_{d}$ & 0.015 min $^{-1}$ \\
$\eta(T)$ & 10 molecule.min $^{-1} @ T_{1} \& 60 @ T_{2}$ \\
$\alpha_{0}$ & 0.03 min $^{-1}$ \\
$\alpha_{s}$ & 3 min $^{-1}$ \\
$K_{s}$ & 0.05 molecule $^{-1}$ \\
$K_{u}$ & 0.0254 molecule $^{-1}$ \\
$K(T)$ & 40 min $^{-1} @ T_{1} \& 80 \mathrm{~min}^{-1} @ T_{2}$ \\
$K_{\text {fold }}$ & $6000 \mathrm{~min}^{-1}$ \\
$P_{t}$ & $2 \times 10^{6}$ molecules \\
\hline
\end{tabular}

[11], [59], [60]. Specifically, the dynamics described above were modeled using differential rate equations, generating a set of 31 differential-algebraic equations (DAEs) of the form

$$
\begin{aligned}
\dot{X}(t) & =F(t ; X ; Y) \\
0 & =G(t ; X ; Y)
\end{aligned}
$$

where $X$ is an 11-dimensional vector whose elements are the differential variables and $Y$ is a 20-dimensional vector whose elements are algebraic variables. This form is known as a semi-explicit DAE. The model possesses 27 kinetic rate parameters. Subsequently, a reduced order model was derived using insight into the system's architecture and separation principles in time and concentrations. As in the full model, this reduced model involves the dynamics of the basic building blocks of the HS response, namely, the $\sigma$ factor $(S)$, the chaperones $(D)$, and the protein folding mechanism. The model equations are as follows:

$$
\begin{aligned}
\frac{d D_{t}}{d t} & =K_{d} S_{f}-\alpha_{d} D_{t} \\
\frac{d S_{t}}{d t} & =\eta(T)-\alpha_{0} S_{t}-\alpha_{s} S: D \\
\frac{d U_{f}}{d t} & =K(T) P_{\text {folded }}-K_{\text {fold }} U: D \\
S: D & =K_{s} \cdot S_{f} \cdot D_{f} \\
U: D & =K_{u} \cdot U_{f} \cdot D_{f} \\
D_{t} & =D_{f}+U: D+S: D \\
S_{t} & =S_{f}+S: D \\
P_{t} & =P_{\text {folded }}+U_{f}+U: D
\end{aligned}
$$

where $U: D$ is the complex formed by the binding of the unfolded proteins $U_{f}$ to $D, S: D$ is the complex formed by the binding of $S$ to $D$, and $P_{t}$ is the total number of proteins in the cell, considered here to be constant. The parameters used in this model are given in Table 1. We replace the algebraic constraints into the initial system (34), then use the facts that $S_{t} \ll D_{t}$ and that $U_{f} \gg 1$ in the wild type bacterial HS response and simplify the expression for $S_{f}$ and $D_{f}$. Simple algebraic manipulations yield a compact description for the reduced order HS model

$$
\begin{aligned}
\frac{d D_{t}}{d t} & =f_{1}\left(D_{t}, U_{f}, S_{t}\right)-\alpha_{d} D_{t} \\
\frac{d S_{t}}{d t} & =\eta(T)-\alpha_{0} \cdot S_{t}-f_{2}\left(D_{t}, U_{f}, S_{t}\right) \\
\frac{d U_{f}}{d t} & =K(T)\left[P_{t}-U_{f}\right]-\left[K(T)+K_{\text {fold }}\right] D_{t} .
\end{aligned}
$$

As in the original equations, the feedforward control is achieved by the temperature dependent function $\eta(T)$ in the ODE describing the dynamics of $S_{t} . f_{1}\left(D_{t}, U_{f}, S_{t}\right)=$ $K_{d}\left(S_{t} /\left(1+\left(K_{s} D_{t} /\left(1+K_{u} U_{f}\right)\right)\right)\right.$ and $f_{2}\left(D_{t}, U_{f}, S_{t}\right)=$ $\alpha_{s}\left(K_{s} D_{t} /\left(1+K_{u} U_{f}\right)\right) /\left(1+\left(K_{s} D_{t} /\left(1+K_{u} U_{f}\right)\right)\right) S_{t}$ describe the various feedback strategies implemented in the HS response. $f_{1}$ is the effect of the sequestration of $S$ by $D$ on $D$ formation, while $f_{2}$ reflects the effect of the regulated degradation of $S$ through the action of the sequestration itself. The dynamics of the third state $U_{f}$ are much faster than those of $S_{t}$ and $D_{t}$. Such stiffness is also strongly present in the full model and creates ill-conditioning and algorithms that do not exploit stiffness are almost certainly doomed to suffer from it. However, stiffness can also be exploited to robustly produce simplified models by singular perturbation, as was done in deriving the 3-state from the full model. By further setting $d U_{f} / d t=0$ to obtain a quasi-steady state approximation, the third equation is then replaced by an algebraic one, and the result is again a DAE. The validity of this approximation has been verified by simulation which showed virtually no difference in the solution of the ODE as compared to that of the DAE. A time course for $\sigma^{32}$ and the level of unfolded proteins is shown in Fig. 3.

3) Results for Robustness Analysis of the HS System: For the HS model, we can consider the problem of proving robust stability for the system under parametric uncertainty. We proceed by nondimensionalizing the states of (35) by their equilibrium values $\left(D_{t_{0}}, S_{t_{0}}, U_{f_{0}}\right)$, followed by a shifting of the equilibrium of the system to the origin. We then obtain a system with states $\left(x_{1}, x_{2}, x_{3}\right)$ that is better conditioned, in the sense that the states are of the same order of magnitude

$$
\begin{aligned}
\frac{d x_{1}}{d t} & =\tilde{f}_{1}\left(x_{1}, x_{2}, x_{3}\right)-\alpha_{d} x_{1} \\
\frac{d x_{2}}{d t} & =\tilde{\eta}(T)-\alpha_{0} x_{2}-\tilde{f}_{2}\left(x_{1}, x_{2}, x_{3}\right) \\
\frac{d x_{3}}{d t} & =K(T)\left[\tilde{P}_{t}-x_{3}\right]-K_{\operatorname{Tot}} x_{1}
\end{aligned}
$$

with $\tilde{f}_{1}\left(x_{1}, x_{2}, x_{3}\right)=\tilde{K}_{d}\left(x_{2} /\left(1+\left(\tilde{K}_{s} x_{1} /\left(1+\tilde{K}_{u} x_{3}\right)\right)\right)\right)$ and $\tilde{f}_{2}\left(x_{1}, x_{2}, x_{3}\right)=\alpha_{s} x_{2}\left(\tilde{K}_{s} x_{1} /\left(1+\tilde{K}_{u} x_{3}\right) /(1+\right.$ $\left.\left.\left(\tilde{K}_{s} x_{1} /\left(1+\tilde{K}_{u} x_{3}\right)\right)\right)\right)$, and where $\tilde{K}_{d}=K_{d} S_{t_{0}} / D_{t_{0}}$, $\tilde{K}_{s}=K_{s} D_{t_{0}}, \tilde{K}_{u}=K_{u} U_{f_{0}}, \tilde{\eta}=\eta / S_{t_{0}}, \tilde{P}_{t}=P_{t} / U_{f_{0}}$ and $K_{\text {Tot }}=D_{t_{0}}\left(K(T)+K_{\text {fold }}\right) / U_{f_{0}}$. We then use $d x_{3} / d t=0$ to get a 2-D state-space $\left(x_{1}, x_{2}\right)$. To proceed, we first define 


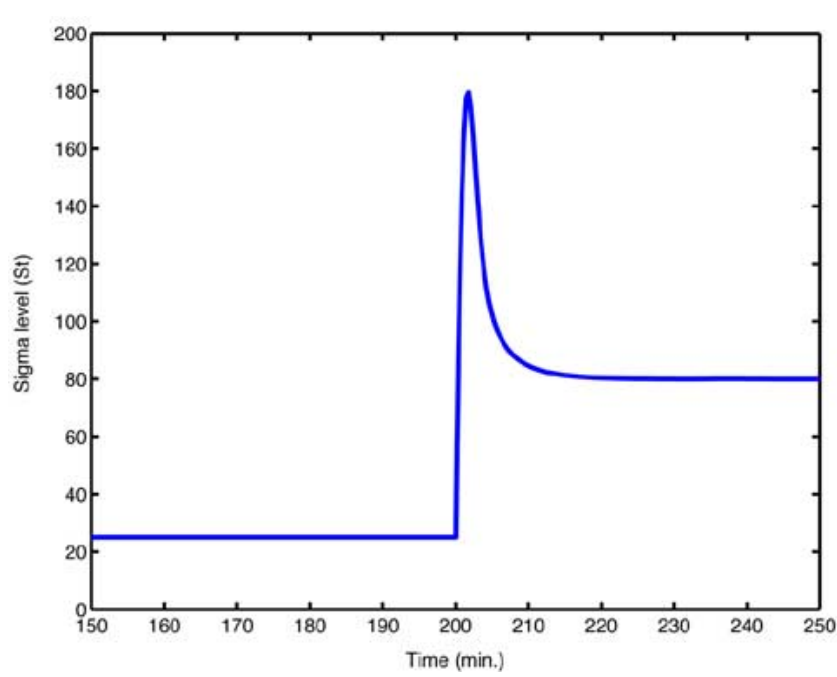

Fig. 3. Plots of: (a) $\sigma^{32}$ and (b) unfolded proteins in the HS response system.

the region $\mathcal{D}$ in the state-space where a Lyapunov function is to be constructed

$$
\mathcal{D}=\left\{x_{i} \in \mathbb{R}:\left(x_{i}-x_{i_{0}}\right)^{2}-\gamma_{i}^{2} \leq 0, i=1,2\right\}
$$

with $\gamma_{i}=0.2, x_{i_{0}}$ denoting the equilibrium of the $i$ th state. For robust stability analysis purposes, we pick two important parameters, $\tilde{\eta}$ and $\alpha_{s}$. $\tilde{\eta}$ depicts the feedforward gain, while $\alpha_{s}$ forms part of the feedback gain. We ask whether the equilibrium of the system described by (35) is stable for all values of $\tilde{\eta}$ and $\alpha_{s}$ in a certain range for $T=T_{1}$

$$
\begin{aligned}
& P=\left\{\tilde{\eta}, \alpha_{s} \in \mathbb{R}^{2}:\left(\tilde{\eta}-\tilde{\eta}_{0}\right)^{2}-\left(\gamma_{3} \tilde{\eta}_{0}\right)^{2} \leq 0,\right. \\
&\left.\left(\alpha_{s}-\alpha_{s_{0}}\right)^{2}-\left(\gamma_{4} \alpha_{s_{0}}\right)^{2} \leq 0\right\}
\end{aligned}
$$

with $\gamma_{3}$ and $\gamma_{4}$ measuring the percentage variation. As these parameters change, the equilibrium of the system also changes. Therefore, in order to fix the equilibrium at the origin, we impose two equality constraints of the form

$$
\begin{aligned}
\alpha_{d} \alpha_{s} \tilde{K}_{s} x_{1_{0}}^{2}-\left[\tilde{K}_{d}\right. & \left(\tilde{\eta}\left(T_{1}\right)-\alpha_{0} x_{2_{0}}\right) \\
& \left.\left.\times\left(1+\tilde{K}_{u}\left(\tilde{P}_{t}-\frac{K_{\text {Tot }}}{K\left(T_{1}\right)} x_{1_{0}}\right)\right)\right)\right]=0
\end{aligned}
$$

and

$$
\tilde{K}_{s} \tilde{K}_{d}\left(\tilde{\eta}\left(T_{1}\right)-\alpha_{0} x_{2_{0}}\right)-\alpha_{s} \tilde{K}_{s}\left(\tilde{K}_{d} x_{2_{0}}-\alpha_{d} x_{1_{0}}\right)=0 .
$$

Note that the vector field is rational, but this case can be treated using Theorem 8 by multiplying out by the (nonvanishing) common denominator of the vector field. Robust stability analysis is then carried out by constructing a parameter-dependent Lyapunov function, using the results in

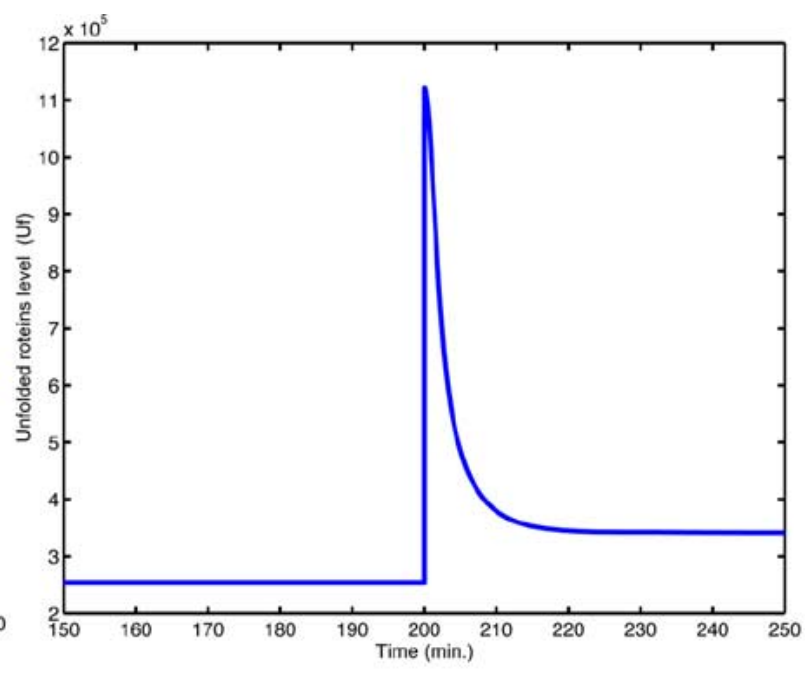

proposition 9 and SOSTOOLS. We start with a quadratic Lyapunov function $V$ that is not parameterized by any parameters; in this case, we could prove stability for $\gamma_{3}=$ $\gamma_{4}=0.45$. When the Lyapunov function is parameterized by $\alpha_{s}$ and $\tilde{\eta}$, we could construct a Lyapunov function for $\gamma_{3}=\gamma_{4}=0.53$. By increasing the complexity of the certificate, we could construct a Lyapunov function for a larger parameter range. In this case, while the equilibrium is stable for even larger parameter sets, the other equilibrium in the system (which is unstable) approaches the equilibrium of interest. Therefore, to prove stability for a larger parameter set, we need to reduce the size of the region $D$ and increase the order of the Lyapunov function. Fig. 4 shows the level curves of the Lyapunov function for two sets of parameters in a parameter set with $\gamma_{3}=\gamma_{4}=0.53$.

\section{Validation/Invalidation of the Model of Heat Shock Response in E. coli}

The new methodology in conjunction with SOSTOOLS can be used to address the critical issue of model validation/invalidation in biological modeling. The key ideas of this methodology can be illustrated in the context of the HS example, where at least two feedback loops are involved in the regulation scheme. We will show rigorously that each loop adds its own important function to the overall system and that both are necessary to explain the phenotypic behavior of the HS system. In previous work, we have used sensitivity analysis and confirmed that these feedback loops indeed increase the robustness to parametric uncertainty [59]. However, upon disabling the degradation (FtsH) feedback loop, one observes in simulation that the transient response to a temperature increase becomes considerably slower. Achieving a faster transient response in the absence of this (FtsH) feedback loop necessitates a substantial increase in the protein synthesis rate, and therefore, produces a larger number of chaperones. Therefore, it is reasonable to conjecture that the (FtsH) feedback loop is instrumental in achieving a fast response to the heat disturbance while using a relatively modest number of chaperones. 

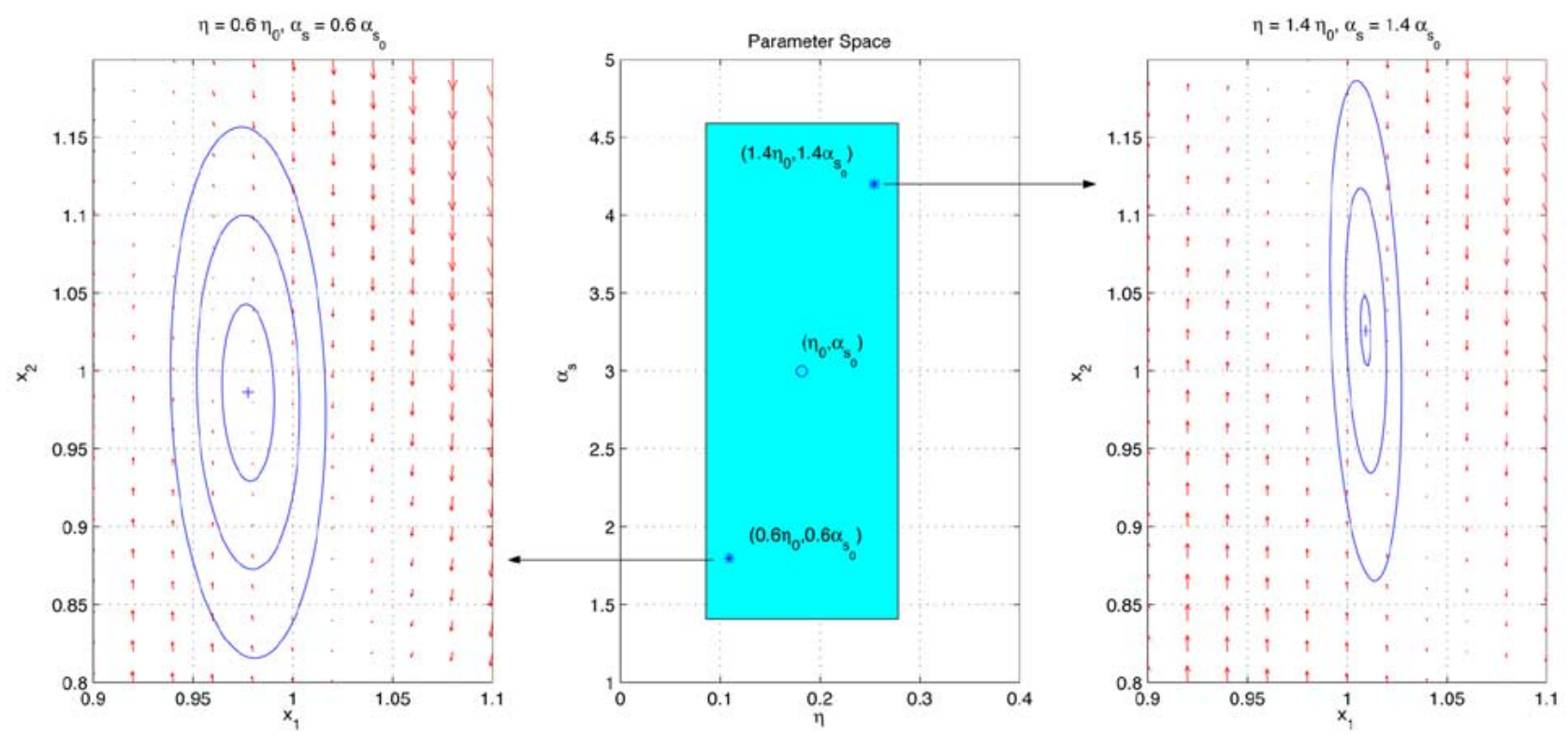

Fig. 4. Robust stability of the model of HS in E. coli.

To actually prove such a conjecture using the invalidation scheme in Section II-B, we will generate some "data" using the model with the degradation (FtsH) loop (35), and compare it to a hypothesized model lacking this feedback. If we denote the state variables $\left(D_{t}, S_{t}, U_{f}\right)$ by $\left(x_{1}, x_{2}, x_{3}\right)$, then the hypothesized model will just be $\dot{x}=f(x, p)$, where the vector field are defined by (35), without the degradation loop. The parameters $p$ will be defined below. A numerical experiment (i.e., a simulation) with the full system is performed, with the parameters fixed at the nominal values. We observe that the corresponding system trajectory satisfies $x(0) \in X_{0}$ and $x(25) \in X_{T}$, where

$$
\begin{aligned}
X_{0}=\{ & \left(x_{1}, x_{2}, x_{3}\right) \in \mathbb{R}^{3}: 0.9 D_{0} \leq x_{1} \leq 1.5 D_{0} \\
& \left.0.9 S_{0} \leq x_{2} \leq 1.5 S_{0}, 2.9 U_{0} \leq x_{3} \leq 3.1 U_{0}\right\}(39) \\
X_{T}=\{ & \left(x_{1}, x_{2}, x_{3}\right) \in \mathbb{R}^{3}: 1.5 D_{0} \leq x_{1} \leq 2.5 D_{0} \\
& \left.2 S_{0} \leq x_{2} \leq 3 S_{0}, 0.5 U_{0} \leq x_{3} \leq 1.5 U_{0}\right\}
\end{aligned}
$$

with $D_{0}, S_{0}$, and $U_{0}$ denoting their steady-state values at low temperature. Note that we use intervals here to take into account the effects of measurement uncertainty, variation of initial conditions, and so on. In addition, we also observe that between time $t=0$ and $t=25$, the state variables satisfy $x(t) \in X$, with

$$
\begin{array}{r}
X=\left\{\left(x_{1}, x_{2}, x_{3}\right) \in \mathbb{R}^{3}: 0.9 D_{0} \leq x_{1} \leq 2.5 D_{0},\right. \\
\left.0.9 S_{0} \leq x_{2} \leq 8 S_{0}, 0.2 U_{0} \leq x_{3} \leq 4 U_{0}\right\} .
\end{array}
$$

For the hypothesized model, we will focus on three parameters $p=\left(K_{d}, \alpha_{0}, \eta(T)\right)$, and assume that the rest are fixed at the nominal values. Plausible ranges for these parameters define the parameter set $P$

$$
\begin{aligned}
& P=\left\{\left(K_{d}, \alpha_{0}, \eta(T)\right) \in \mathbb{R}^{3}: 0.5 \bar{K}_{d} \leq K_{d} \leq 5 \bar{K}_{d},\right. \\
& \left.0.5 \bar{\alpha}_{0} \leq \alpha_{0} \leq 1.5 \bar{\alpha}_{0}, 0.5 \overline{\eta(T)} \leq \eta(T) \leq 1.5 \overline{\eta(T)}\right\}
\end{aligned}
$$

where $\bar{K}_{d}, \bar{\alpha}_{0}$, and $\overline{\eta(T)}$ denote their nominal values. We deliberately make the upper bound for $K_{d}$ quite large, since one obvious way for obtaining a fast response is to increase the number of chaperones, corresponding to increasing this parameter. With our method, we can find a barrier certificate for these model and data

$$
\begin{aligned}
B(x, t)= & 1.4233 t-4.4566 x_{2}-.0051737 x_{3} \\
& +.021541 x_{1} t x_{3}+.016599 t x_{1}^{2}+.020129 t x_{2}^{2} \\
& -.081828 x_{1} t-.054129 x_{3} t-.71041 x_{2} t \\
& +.54734 \times 10^{-4} x_{3} t x_{2}+.0022033 x_{1} t x_{2} \\
& +.0067639 x_{1}-.76233 \times 10^{-3} x_{3} x_{2} \\
& +.0010018 x_{3} x_{1}+.0052243 t^{2} x_{1}+.02375 t^{2} x_{2} \\
& -.66663 \times 10^{-3} t^{2} x_{3}-.68942 \times 10^{-3} t^{2} x_{1}^{2} \\
& -.0024426 t^{2} x_{2}^{2}+.74858 \times 10^{-4} t^{2} x_{3}^{2} \\
& +.0029471 t^{2}+.95341 \times 10^{-3} x_{1}^{2}+.010339 x_{2}^{2} \\
& +.27438 \times 10^{-3} x_{1} t^{2} x_{3}-.40973 \times 10^{-5} t^{2} x_{3} x_{2} \\
& -.79472 \times 10^{-3} t^{2} x_{1} x_{2}-.015999 x_{2} x_{1} \\
& +.0052841 x_{3}^{2} t+.0019683 x_{3}^{2}
\end{aligned}
$$

in effect proving that the model without the degradation (FtsH) loop and with parameters $K_{d}, \alpha_{0}, \eta(T)$ satisfying (42) cannot possibly generate a time response that satisfies (39)-(41). This indicates that an inherent mechanism is missing from this model. When the FtsH mechanism is 
included, obviously there are values for parameters $K_{d}, \alpha_{0}$, and $\eta(T)$ (for example, we can simply choose the nominal values $\bar{K}_{d}, \bar{\alpha}_{0}$, and $\overline{\eta(T)}$ ) such that the model has a time response that satisfies (39)-(41).

\section{Stochastic Reachability Analysis of the Bacteriophage Lambda}

One of the best studied examples of multistability in genetic systems is the bacteriophage $\lambda$ system [18], [21]. This system has largely been used as a prototype for the investigation of stochasticity in cellular networks, and an illustration of the mathematical challenges that reside in such investigations. Phages are viral organisms that can either be in the lysogenic (latent) or lytic (active) state. If following its infection of $E$. coli, the $\lambda$-phage virus enters the lysogenic pathway, it represses its own developmental functions and integrates its DNA into the host chromosome. Otherwise, it enters the lytic pathway and is active. The dynamics of the transcriptional network underlying the formation of these states are very complex, and have been thoroughly studied [21], [61]. Instead of describing the full complexity of the $\lambda$-phage system, we limit ourselves here to a simplified model of the process that still captures the essence of its exhibited bistability, in addition to the interplay between noise and dynamics in its operation.

1) The $\lambda$-Phage One-Dimensional Model: The representative simple model we adopt was first discussed by Hasty et al. [62], and is intended to be a minimal model that captures the bistable nature of the $\lambda$ system. The model describes the dynamics of the CI protein, the product of the cI gene. CI, acting as a dimer, can regulate its own synthesis by binding to the cI gene promoter region $\mathrm{OR}_{2}$ and increasing transcription or to gene promoter $\mathrm{OR}_{3}$ and repressing transcription. It can also bind to a third gene promoter $\mathrm{OR}_{1}$, which we ignore in this model for simplicity. We assume the fast binding reactions (such as binding and dissociation) to be in equilibrium with respect to the slow reactions (such as protein synthesis and degradation). Under this assumption, and following [62], we let $X, X_{2}$, and $D$ denote the repressor, repressor dimer, and DNA promoter site, respectively. Then, the equilibrium reactions can be written as

$$
\begin{aligned}
& 2 X \stackrel{K_{1}}{\rightleftharpoons} X_{2}, \quad D+X_{2} \stackrel{K_{2}}{\rightleftharpoons} D X_{2}, \\
& D+X_{2} \stackrel{K_{3}}{\rightleftharpoons} D X_{2}^{*}, \quad D X_{2}+X_{2} \stackrel{K_{4}}{\rightleftharpoons} D X_{2} X_{2}
\end{aligned}
$$

where the $D X_{2}$ and $D X_{2}^{*}$ complexes denote binding to the $\mathrm{OR}_{2}$ or $\mathrm{OR}_{3}$ sites, respectively, $D X_{2} X_{2}$ denotes binding to both sites, and the $K_{i}$ are forward equilibrium constants. We further set $K_{3} \simeq K_{2}$ and $K_{4} \simeq 5 K_{2}$.

The slow reactions are transcription and degradation. At first, we assume that translation is a fast process and therefore lump its dynamics with those of transcription. Later, we will relax this assumption in order to construct a higher order model of the system. The molecular reactions describing the slow dynamics are given by

$$
D X_{2}+P \stackrel{k_{t}}{\longrightarrow} D X_{2}+P+n X, \quad X \stackrel{k_{d}}{\longrightarrow} A
$$

where $P$ denotes the concentration of RNA polymerase, and $n$ is the number of proteins per mRNA transcript. To model the system, we state variables as $x=[X], y=\left[X_{2}\right], d=$ $[D], u=\left[D X_{2}\right], v=\left[D X_{2}^{*}\right]$, and $z=\left[D X_{2} X_{2}\right]$, where [.] denotes concentration.

The evolution of the concentration of the repressor $x$ can then be described by

$$
\dot{x}=-2 k_{1} x^{2}+2 k_{-1} y+n k_{t} p_{0} u-k_{d} x+q .
$$

Here, the concentration of RNA polymerase $p_{0}$ is assumed to remain constant during time. The parameter $q$ is the basal rate of production of CI, i.e., the expression rate of the cI gene in the absence of a transcription factor. Because the reactions in (43) are fast, $y, u$, and $d$ have algebraic expressions in terms of $x$. We further use the fact that total amount of DNA promoter sites is constant at a value $d_{T}$ to derive an expression of $d$ in terms of $x$. The resulting one-dimensional model for $x$ is then given by

$$
\dot{x}=\frac{n k_{t} p_{0} d_{t} K_{1} K_{2} x^{2}}{1+2 K_{1} K_{2} x^{2}+5 K_{1}^{2} K_{2}^{2} x^{4}}-k_{d} x+q .
$$

To reduce the size of the parameter space, we eliminate two of the parameters in (45) by rescaling the repressor concentration $x$ and time. To this end, we define the dimensionless variables $\tilde{x}=x \sqrt{K_{1} K_{2}}$ and $\tilde{t}=t\left(q \sqrt{K_{1} K_{2}}\right)$. On substitution into (45), we obtain

$$
\dot{x}=\frac{\alpha x^{2}}{1+2 x^{2}+5 x^{4}}-\gamma x+1
$$

where the tildes have been suppressed, and we have defined $\alpha=n k_{t} p_{0} d_{T} / q$ and $\gamma=k_{d} /\left(q \sqrt{K_{1} K_{2}}\right)$.

For this equation, there are two types of behavior, depending on the choice of the parameters $\alpha$ and $\gamma$. As the parameters vary, the number of equilibria in the system changes from one to three and then back to one. When there is only one stable equilibrium and all concentrations evolve to that equilibrium. When there are three equilibria, two of which are stable and the other unstable. Therefore, the asymptotic behavior of the system is dependent on the initial condition. Such a behavior is known as bistable and appears frequently in many areas of engineering and physics.

2) Stochastic Reachability Analysis of the One-Dimensional SDE Formulation of Phage $\lambda$ : Although a given trajectory of the phage $\lambda$ system will converge to one of the two equilibria at steady state, it might leave this steady state and switch to the other one under the action of the biochemical 


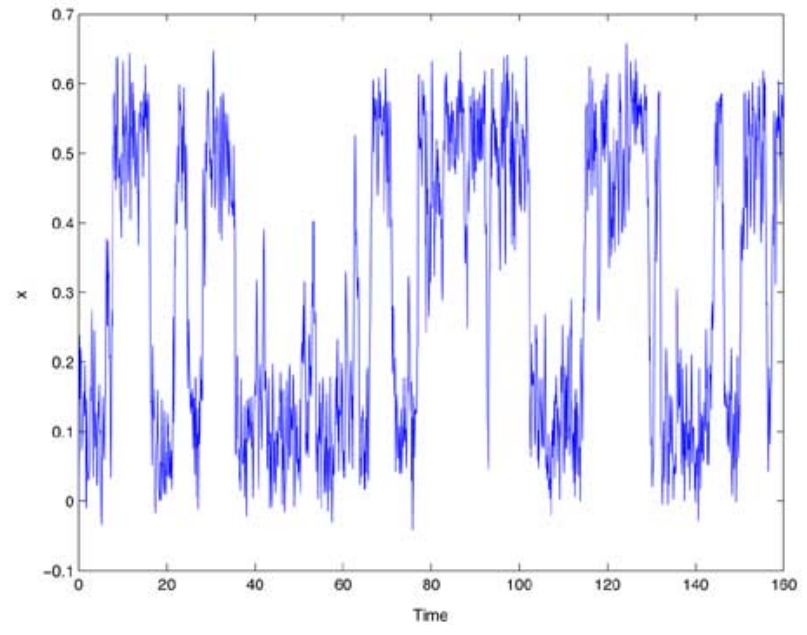

Fig. 5. Switching between equilibria in the $\lambda$ system caused by noise.

noise affecting the many reactions occurring in the system. We show this behavior in Fig. 5 where a realization based on the stochastic simulation algorithm of Gillespie is shown. In this section, we are concerned with the characterization of the statistics of such switching events.

However, here we consider a Langevin SDE formulation of the $\lambda$-phage system. To do that, we start by incorporating additive noise in the deterministic rate equation of (46). If we take the dynamical variable $x$ to represent the repressor number within a colony of cells, we can crudely think of an additive white noise term as a randomly varying external field acting on the biochemical reactions, hence accounting for the impact of the environment on the system. The resulting SDE is given by

$$
d x(t)=f(x) d t+\sigma d \xi(t)
$$

where $f(x)$ is the right-hand side of (46), $\xi(t)$ is a Wiener process, and $\sigma$ is a scalar. The presence of this noise source poses new questions. For example, while in the deterministic description trajectories that reach either steady state come to rest there, in the stochastic case they never do so because of noise. One can then ask whether there is a large probability of noise-induced switching between equilibria within a given time frame, then describe its correlation with the noise intensity affecting the system. These quantities can be computed through the exact analytical solution of the SDE. However, when such solution cannot be computed explicitly as it is often the case, statistics determined from extensive simulations are usually used. Alternatively here, we propose that the sum of squares and SOSTOOLS machinery can be used as an efficient algorithmic method for such investigations. We specifically illustrate this point by investigating the situation where the model in (46) is started close to one of the two stable equilibria, and an estimate of the the probability of transition to a region around the other stable equilibrium is computed. We will refer to this as the bound on the probability of false switching caused by noise. For example, the setup for this problem is shown in Fig. 6 where we are interested in whether an initial trajectory from a point inside a set of variable size $r$ centered at the high equilibrium

$$
\chi_{0}=\left\{x \in \mathbb{R} \mid(x-0.56)^{2}-r^{2} \leq 0\right\}
$$

can ever reach a region around the other equilibrium,

$$
\chi_{u}=\left\{x \in \mathbb{R} \mid(x-0.098)^{2}-0.02^{2} \leq 0\right\}
$$

when the total state-space of interest is

$$
\chi=\{x \in \mathbb{R} \mid x(x-1) \leq 0\} .
$$

This question, however, is ill-posed if the time horizon over which this probability is to be computed is not bounded. Indeed, the way the noise enters in (47) means that if the state-space $\mathcal{X}$ is large enough (say, the whole real line) and the time is infinite, the probability of reaching every point in the state-space should be one.

A more meaningful biological question that our methods can answer is the following. Starting from a region around one equilibrium, estimate the probability of reaching the other equilibrium in a finite time horizon, say from $t=0$ to two nondimensional time units. Time is now another variable in the system, and instead of constructing a time-independent $B(x)$, we construct a time-dependent $B(x, t)$ to estimate this probability. We also increase the state-space adequately, so that the "escape" probability is reduced significantly. As $\sigma$ is varied in this modified problem, our methodology gives the following results, which we compare with probability estimates obtained by direct simulations of the stochastic differential equation in (47). When $\sigma=1$, $98 \%$ of the simulations enter the region around the other equilibrium, whereas our methodology gives an upper bound of $\beta=0.99$. When $\sigma=0.5$, direct simulations gives 0.57 , and our methodology returns $\beta=0.6$. We see now that the expected result is obtained; as $\sigma$ is decreased, the probability of reaching the other equilibrium (false switching) is decreased, and the upper bounds are close to the probability estimates obtained by direct simulations.

Using the same methodology, we can also address the dependence of the switching probability from one steady state to the other on time and for a given noise intensity. We show the results of our computations in Fig. 7 where the upper bound on this probability is plotted as a function of time, along with estimates of the probability, obtained from simulations of the SDE. It can be seen that the two plots are satisfactorily close to each other. However, while we had to run a large number of simulations (of order a thousand) to compute estimates of the probability, with the computational effort increasing as the simulation time interval was increased, the barrier technique generated good upper bounds 


$$
d x=\left(\frac{50 x^{2}}{1+2 x^{2}+5 x^{4}}-15 x+1\right) d t+d \xi(t)
$$

$$
\begin{array}{ll}
x_{1}^{*}=0.560 & \text { (stable) } \\
x_{2}^{*}=0.098 \text { (stable) } \\
x_{3}^{*}=0.255 \text { (unstable) }
\end{array}
$$

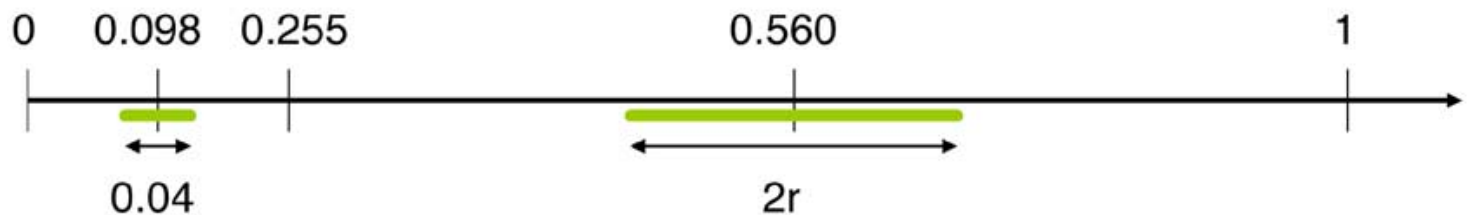

$$
\begin{aligned}
X_{u} & =\left\{x \in R \mid(x-0.098)^{2}-0.02^{2} \leq 0\right\} \\
X_{0} & =\left\{x \in R \mid(x-0.56)^{2}-r^{2} \leq 0\right\} \\
X & =\{x \in R \mid x(x-1) \leq 0\}
\end{aligned}
$$

Fig. 6. Setup for computing the bound of the probability of false switching because of noise, for system (47).

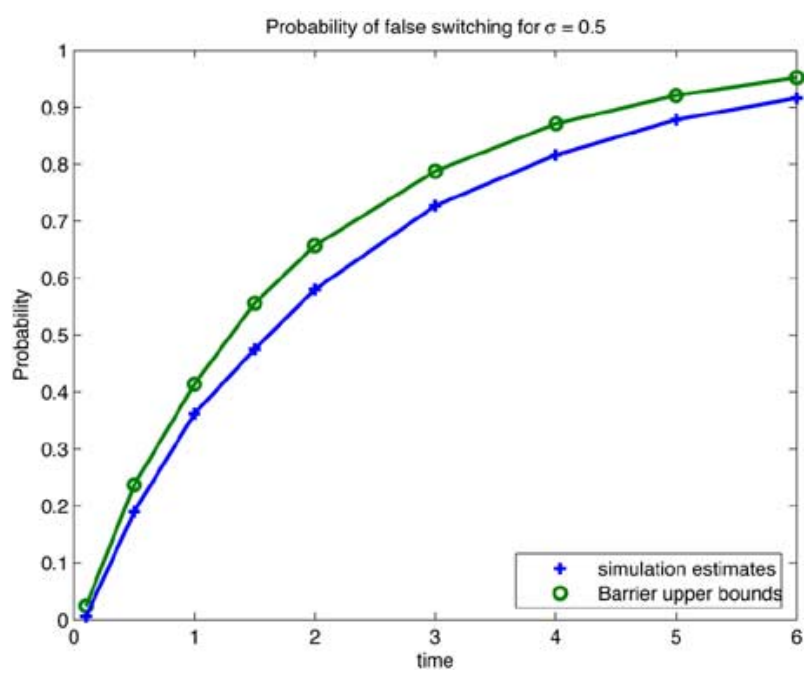

Fig. 7. Probability of false switching from the high steady to the low steady state as a function of time under the influence of noise. The blue line depicts probabilities computed through the use of barrier functions while the green line depicts the probabilities computed from 2000 realizations obtained from direct simulations of the SDE.

in a fraction of time and each run had the same computational burden. This will become increasingly important as we consider larger systems where exact simulations become prohibitively slow. In general, probabilities computed using our barrier methods are conservative bounds on the true probabilities. However, as illustrated in our example, these bounds can yield accurate results.

The same techniques can again be used to compute probability lower bounds. For example, Fig. 8 shows the upper and lower bounds for the probability of escaping a neighborhood

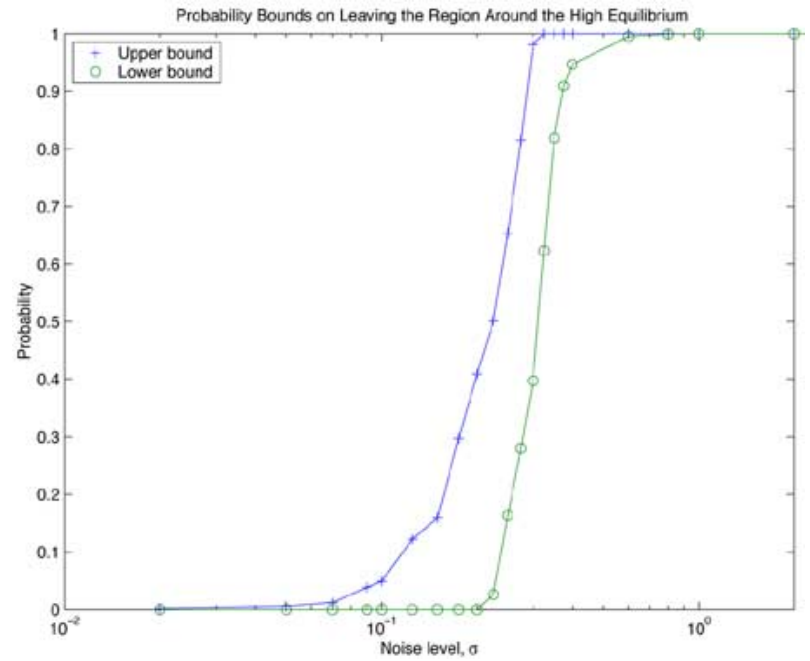

Fig. 8. Upper and lower bounds on probability of escaping a neighborhood of the high equilibrium, computed using the Barrier technique.

of the high equilibrium in a finite time horizon as the noise intensity increases. It is interesting to note that the probability goes sharply from zero to one (indicating a phase transition) as the noise is increased, and that the technique we propose can be used to estimate the critical noise intensity necessary for escaping the given region around the equilibrium.

3) The $\lambda$-Phage Two-Dimensional Model: The model we have presented in the previous section is particularly simple. In order to demonstrate the capabilities of the methodology in higher dimensional systems, we propose to adopt a 2-D description of the $\lambda$ system while giving a more plausible description of the biochemical noise affecting the system. In order to get a rich model description, we start by introducing 
a crucial step that was eliminated from the model to reduce it to a 1-D description: translation. Recall that in the previous model, the production of $X$ follows

$$
D X_{2}+P \stackrel{k_{t}}{\longrightarrow} D X_{2}+P+n X, \quad X \stackrel{k_{d}}{\longrightarrow} A .
$$

This means that $X$ is produced in a single-step process, then degraded. More realistically, it should be produced through a two step process of transcription first, then translation. The resulting description is

$$
\begin{gathered}
D X_{2}+P \stackrel{k_{t}}{\longrightarrow} D X_{2}+P+M, \quad M \stackrel{k_{T}}{\longrightarrow} M+n X, \\
M \stackrel{k_{d}}{\longrightarrow} B, \quad X \stackrel{k_{m}}{\longrightarrow} A
\end{gathered}
$$

and the resulting 2-D model is

$$
\begin{aligned}
\dot{m} & =k_{t} p_{0} u-k_{d} m+q \\
\dot{x} & =n k_{T} m-k_{m} x+2 k_{-1} y-2 k_{1} x^{2} .
\end{aligned}
$$

Proceeding as before, we eliminate $u$ from the equation to get

$$
\begin{aligned}
\dot{m} & =\frac{k_{t} p_{0} d_{t} K_{1} K_{2} x^{2}}{1+2 K_{1} K_{2} x^{2}+5 K_{1}^{2} K_{2}^{2} x^{4}}-k_{d} m+q \\
\dot{x} & =n k_{T} m-k_{m} x .
\end{aligned}
$$

Carrying the change of variables $\tilde{x}=x \sqrt{K_{1} K_{2}}$, $\tilde{m}=m \sqrt{K_{1} K_{2}}$, and $\tau=t\left(q \sqrt{K_{1} K_{2}}\right)$, we get

$$
\begin{aligned}
\dot{\tilde{m}} & =\frac{\alpha \tilde{x}^{2}}{1+2 \tilde{x}^{2}+5 \tilde{x}^{4}}-\gamma \tilde{m}+1 \\
\dot{\tilde{x}} & =\frac{n k_{T}}{q \sqrt{K_{1} K_{2}}} \tilde{m}-\frac{k_{m}}{q \sqrt{K_{1} K_{2}}} \tilde{x}
\end{aligned}
$$

where $\alpha=k_{t} p_{0} d_{t} / q$ and $\gamma=k_{d} / q \sqrt{K_{1} K_{2}}$. Following [62], we fix the numerical values of the parameters in the system so as to get the following model:

$$
\begin{aligned}
\dot{\tilde{m}} & =\frac{50 \tilde{x}^{2}}{1+2 \tilde{x}^{2}+5 \tilde{x}^{4}}-150 \tilde{m}+1 \\
\dot{\tilde{x}} & =10 \tilde{m}-\tilde{x}
\end{aligned}
$$

Note that in the phase plane, the deterministic part of this system results in three equilibria as before, two of which are stable and the other unstable. The phase-plane of system (52), (53) is shown in Fig. 9.

Rather than introducing additive noise in an ad hoc manner to this 2-D system, we consider more biologically plausible sources of noise that originate from intrinsic biochemical fluctuations. However, starting from a detailed account of the

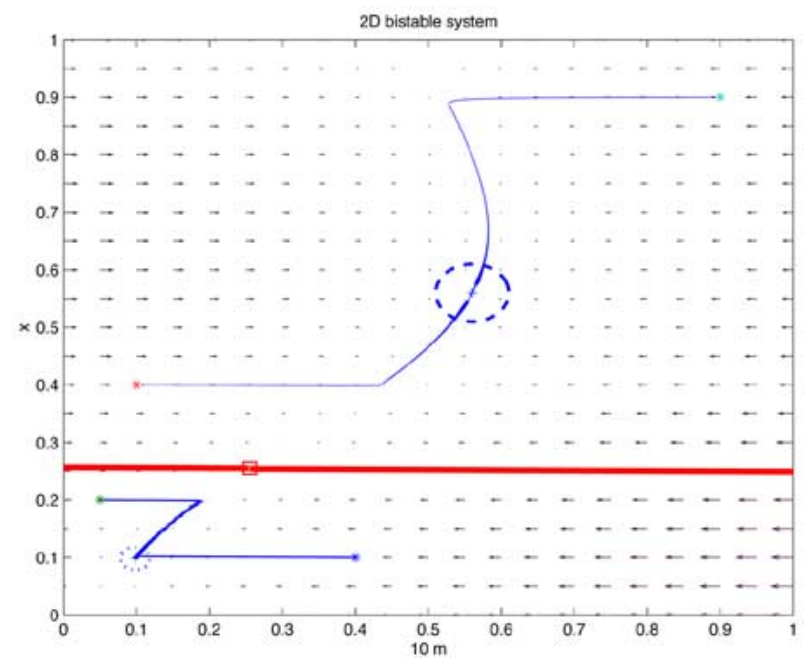

Fig. 9. Phase plane for system (52), (53). Arrows denote the vector field, solid lines are trajectories from initial conditions denoted by “*”. Equilibria are shown by "+" (stable) and " $\square$ " (unstable). The solid thick line is a separatix-it divides the phase plane in two, so that if the deterministic system is initialized in one region then all trajectories flow toward one equilibrium, whereas if the system is initialized in the other all trajectories flow to the other equilibrium.

noise that stems from all the elementary chemical reactions in the network, we carry out two approximations that generate an SDE with multiplicative noise as the system's stochastic description.

For the system under study, the elementary (birth and death) chemical reactions are given by (43) and (51). Each of these elementary reaction steps is characterized by its probability of occurrence. This probability is in turn captured by the so-called propensity functions, which are simple functions of the rate constants and concentration of the reactants. The description based on the elementary reactions and their probability of occurrence generates a continuous-time, discrete-state Markov chain that is often studied through Monte Carlo simulations.

Chemical reactions, including the example that we are considering, often occur at drastically different time scales, therefore making analysis and simulation of the Markov chain description of the processes involved computationally challenging. To circumvent this stiffness, it is common to replace the elementary reaction with the so-called elementary-complex reactions. One elementary-complex reaction is formed by an aggregation of elementary reactions. Therefore, the probability of occurrence of an elementary-complex reaction is an involved function of the state of the system. One method to compute these probabilities is to use Michaelis-Menten and various other well established approximations for the deterministic description of the system, then use the resulting expressions to define the complex reactions and their propensities [63]. For the example considered here, this will translate into replacing the elementary reactions in (43) and (51), by complex birth and death reactions for $\tilde{m}$ and $\tilde{x}$ whose propensities are taken from the differential equations in (52) and (53). 
With these elementary-complex propensities generating a reduced order stochastic description of the $\lambda$-phage system, we carry out a diffusion approximation that transforms the Markov chain type of description of these molecular reactions into a stochastic differential equation. The premises of this procedure were recently reinvestigated in the work of Gillespie [64], to which we refer the reader for details. Here, we only state the final result. If $a_{i}(X)$ are the propensity functions that determine transition probabilities, $A$ is a column vector formed by these propensities, and $S$ is the stoichiometry matrix, then the deterministic description of the system [as in (52) and (53)] is given by

$$
\frac{d X}{d t}=S A(X)
$$

and the SDE generated by the diffusion approximation is given by

$$
d X=S A(X) d t+S \sqrt{\operatorname{diag}(A(X))} d \xi
$$

With the elementary to elementary-complex and diffusion approximations in place, the final CLE describing the 2-D $\lambda$ system becomes

$$
\begin{aligned}
d \tilde{m}= & \left(\frac{50 \tilde{x}^{2}}{1+2 \tilde{x}^{2}+5 \tilde{x}^{4}}-150 \tilde{m}+1\right) d t \\
& +\sqrt{\frac{50 \tilde{x}^{2}}{q\left(1+2 \tilde{x}^{2}+5 \tilde{x}^{4}\right)}} d \xi_{1}+\sqrt{\frac{150 \tilde{m}}{q}} d \xi_{2}+\frac{d \xi_{3}}{\sqrt{q}} \\
d \tilde{x}= & (10 \tilde{m}-\tilde{x}) d t+\sqrt{\frac{10 \tilde{m}}{q}} d \xi_{4}+\sqrt{\frac{\tilde{x}}{q}} d \xi_{5} .
\end{aligned}
$$

For this system, we will again compute a bound on the probability of reaching one of the steady states from the other one during a fixed time. In particular, we consider whether from an initial region around the low equilibrium defined by

$\chi_{0}=\left\{x \in \mathbb{R} \mid(x-0.098)^{2}+(10 m-0.098)^{2}-0.02^{2} \leq 0\right\}$

one can reach a region around the high equilibrium defined by

$$
\chi_{u}=\left\{x \in \mathbb{R} \mid(x-0.560)^{2}+(10 m-0.560)^{2}-0.05^{2} \leq 0\right\}
$$

in a certain time span, say, $t \in[0,10]$ for different noise intensities. In this case, and for a fixed structure of the system, different noise intensities can be achieved by changing the number of molecules in the system. We therefore define $\hat{x}=$ $N x$ for (54). $N$ is commonly called the system size. This change of variables scales the number of molecules without

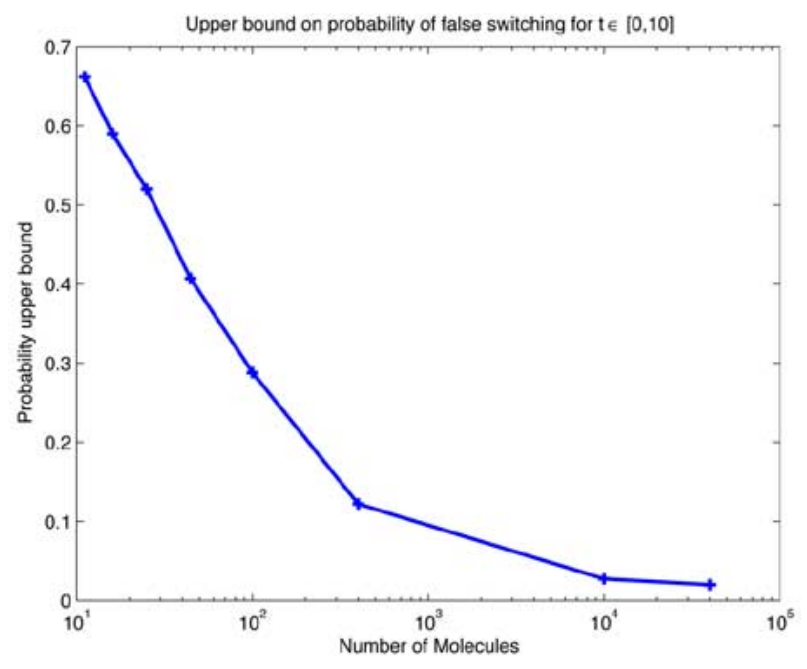

Fig. 10. Upper bound on switching probability from the higher equilibrium as a function of the number of molecules in the system.

modifying the dynamics of the deterministic system. However, the associated noise intensity grows as the square root of $N$. This in agreement with the common intuition [and can also be directly seen from (54)] that as the number of molecules in a system increases, the noise strength affecting it, normalized by the mean, decreases.

With $N$ as our varying noise intensity, we attempt to answer the false switching question. We construct certificates $B(x, t)$ algorithmically, again using the methodology described earlier. Note that the nonpolynomial nature of the vector field (the way noise affects the system is through the square root of some polynomial) does not cause any problems, as these terms appear squared in the expressions to be tested-see condition (32) in Theorem 15. The upper bound on this probability as function of $N$ is given in Fig. 10. Notice the expected fact that as $N$ increase, therefore yielding lower noise affecting the system, the probability of false switching decreases. As in the previous case, this result was obtained without time consuming computations and was not based on numerous simulations of the corresponding SDE.

\section{CONCLUSION}

In this paper, we have illustrated how the sum of squares technique can be used to treat algorithmically a number of issues related to the analysis of nonlinear systems. We specifically approached the problems of model validation/invalidation, robust stability analysis, and stochastic reachability analysis. Although the methods we presented are appropriate for a large number of applications [43], we focused on biological applications where these problems are of central importance.

In the context of robust stability analysis, we illustrated the use of efficient algorithmic procedures to analyze nonlinear systems described by ODEs with parametric uncertainty, and which evolve under equality and inequality constraints. We showed how these inequality constraints arise naturally to describe the region of the parameter space that is of interest, and also how equality constraints are used to account for the 
change in the location of the equilibrium as the parameters change. In this setting, robust stability of the system can be checked by constructing Lyapunov functions using the sum of squares decomposition and SOSTOOLS.

In the context of model validation of continuous-time nonlinear systems with uncertain parameters, we presented methods that employ functions of state-parameter-time, called barrier certificates, whose existence proves that a model and a feasible parameter set are inconsistent with some time-domain experimental data. Construction of barrier certificates can be performed by convex optimization utilizing recent results on the sum of squares decomposition of multivariate polynomials.

While model invalidation and stability analysis where formulated in a deterministic setting, we demonstrated stochastic analysis can be performed for biological examples where accounting for noise is important. Specifically, we used barrier certificates that generate nonnegative super-martingales under the given system dynamics to handle the analysis problem by computing certified upper bounds on the probability of reaching one stable steady state from the other one in a specified time interval, and as a function of the noise intensity.

All the above examples illustrate the successful use of widely promising new methods for the study of old problems. While these methods opened the door to exciting applications, they also uncovered great research challenges that must be overcome to broaden the applicability of these methods and resolve their limitations.

A major challenge that must addressed is the issue of scalability of these methods with respect to model and data. In model invalidation, for example, the complexity grows very fast in the number of data points used. In addition, these methods become computationally inefficient as the state dimension increases, both for barrier and Lyapunov functions. Furthermore, the current output of the model invalidation scheme lacks any feedback from analysis/invalidation to modeling itself. In other terms, if a barrier functions is found, then the model is invalidated but there is no indication as to what part of the model is deficient. An important research direction would involve the modification of these the methods as to give hints to those aspects of the model that need to be refined.

Finally, an important computational challenge is the inefficiency of the algorithmic constructions using sum of squares in problems exhibiting "stiffness" caused by the presence of drastically different time scales. To alleviate this stiffness, some insight can be gained through singular perturbation methods. When applied, for example, to the structures of Lyapunov function, these methods can result in semidefinite programming conditions that are numerically well-conditioned [30]. These issues, and others, are currently the subject of an active research effort.

\section{REFERENCES}

[1] H. Kitano, "Systems biology: A brief overview," Science, vol. 295, no. 5560, pp. 1662-1664, 2002.
[2] H. Wiley, S. Shvartsman, and D. Lauffenburger, "Computational modeling of the EGF-receptor system: A paradigm for systems biology," Trends Cell Biol., vol. 13, no. 1, pp. 43-50, 2003.

[3] T. Ideker, T. Galitski, and L. Hood, "A new approach to decoding life: Systems biology," Annu. Rev. Genomics Hum. Genet., vol. 2, pp. 343-372, 2001.

[4] M. Csete and J. Doyle, "Bow ties, metabolism and disease," Trends Biotechnol., vol. 22, no. 9, pp. 446-450, 2004.

[5] L. Hood, J. Heath, M. Phelps, and B. Lin, "Systems biology and new technologies enable predictive and preventative medicine," Science, vol. 306, no. 5296, pp. 640-643, 2004.

[6] E. Butcher, E. Berg, and E. Kunkel, "Systems biology in drug discovery," Nature Biotechnol., vol. 22, pp. 1253-1259, 2004.

[7] S. Prajna, "Barrier certificates for nonlinear model validation," $\mathrm{Au}$ tomatica, vol. 42, no. 1, pp. 117-126, 2006.

[8] M. Morohashi et al., "Robustness as a measure of plausibility in models of biochemical networks," J. Theor. Biol., vol. 216, no. 1, pp. 19-30, 2002.

[9] M. Csete and J. Doyle, "Reverse engineering of biological complexity," Science, vol. 295, no. 5560, pp. 1664-1669, 2002.

[10] J. Stelling et al., "Robustness of cellular functions," Cell, vol. 118, no. 6, pp. 675-685, 2004.

[11] H. El-Samad, H. Kurata, J. Doyle, C. Gross, and M. Khammash, "Surviving heat shock: Control strategies for robustness and performance," Proc. Nat. Acad. Sci. USA, vol. 102, no. 9, pp. 2736-2741, 2005.

[12] A. Liapunov, "Problème général de la stabilité du mouvement," An. Fac. Sci. Toulouse, vol. 9, pp. 203-474, 1907.

[13] A. Papachristodoulou and S. Prajna, "On the construction of Lyapunov functions using the sum of squares decomposition," in Proc. 41st IEEE Conf. Decision and Control 2002, pp. 3482-3487.

[14] M. Walter et al., "Enhancers increase the probability but not the level of gene expression," Proc. Nat. Acad. Sci. USA, vol. 92, no. 15, pp. 7125-7129, 1995

[15] M. Dingemanse et al., "The expression of liver specific genes within rat embryonic hepatocytes is a discontinuous process," Differentiation, vol. 56, no. 3, pp. 153-162, 1994.

[16] M. Ko, S. Nakauchi, and N. Takahashi, "The dose dependence of glucocorticoid-inducible gene expression results from changes in the number of transcriptionally active templates," $E M B O J$. , vol. 9, no. 9, pp. 2835-2842, 1990.

[17] D. Bennett, "Differentiation in mouse melanoma cells: Initial reversibility and an on-off stochastic model," Cell, vol. 34, no. 2, pp. 445-453, 1983

[18] A. Arkin, J. Ross, and H. McAdams, "Stochastic kinetic analysis of the developmental pathway bifurcation in phage $\lambda$-infected escehrichia coli cells," Genetics, vol. 149, no. 4, pp. 1633-1648, 1998.

[19] H. El-Samad, M. Khammash, L. Petzold, and D. Gillespie, "Stochastic modeling of gene regulatory networks," Int. J. Robust Nonlinear Control, vol. 15, pp. 691-711, 2005.

[20] F. Benzt, "Cell death and cancer therapy," Curr. Opin. Pharmacol., vol. 1, pp. 337-341, 2001.

[21] M. Ptashne, A Genetic Switch: Phage Aand Higher Organisms. Cambridge, MA: Blackwell, 1992.

[22] O. Kobiler et al., "Quantitative analysis of bacteriphage $\lambda$ genetic network," Proc. Nat. Acad. Sci. USA, vol. 102, no. 12, pp. 4470-4475, 2005.

[23] D. T. Gillespie, "A rigorous derivation of the Chemical Master Equation," Physica A, vol. 188, pp. 404-425, 1992.

[24] N. van Kampen, Stochastic Processes in Physics and Chemistry. Amsterdam, The Netherlands: Elsevier Science Publishing Company, 1992.

[25] D. T. Gillespie, "A general method for numerically simulating the stochastic time evolution of coupled chemical reactions," J. Comp. Phys., vol. 22, pp. 403-434, 1976.

[26] — "Exact stochastic simulation of coupled chemical reactions," J. Phys. Chem., vol. 81, pp. 2340-2361, 1977.

[27] M. Rathinam, L. Petzold, Y. Cao, and D. Gillespie, "Stiffness in stochastic chemically reacting systems: The implicit tau-leaping method," J. Chem. Phys., vol. 19, pp. 12 784-12 794, 2003.

[28] L. Pratt, "A statistical method for identifying transition states in high dimensional problems," J. Chemical Physics, vol. 85, no. 9, pp. 5045-5048, 1986. 
[29] R. Radhakrishnan and T. Schlick, "Orchestration of cooperative events in DNA synthesis and repair mechanism unraveled by transition path sampling of dna poly-merase beta's closing," Proc. Nat. Acad. Sci. USA, vol. 101, no. 16, pp. 5970-5975, 2004.

[30] H. K. Khalil, Nonlinear Systems. Englewood Cliffs, NJ: Prentice-Hall, 1996.

[31] V. Zubov, Methods of A. M. Lyapunov and Their Application. Groningen, The Netherlands: Noordhoff, 1964.

[32] W. Hahn, Stability of Motion. New York: Springer-Verlag, 1967.

[33] S. Boyd, L. E. Ghaoui, E. Feron, and V. Balakrishnan, Linear Matrix Inequalities in System and Control Theory. Philadelphia, PA: SIAM, 1994.

[34] L. Vandenberghe and S. Boyd, "Semidefinite programming," SIAM Rev., vol. 38, no. 1, pp. 49-95, 1996.

[35] C. A. Desoer and M. Vidyasagar, Feedback Systems: Input-Output Properties. New York: Academic, 1975.

[36] E. Feron, P. Apkarian, and P. Gahinet, "Analysis and synthesis of robust control systems via parameter-dependent Lyapunov functions," IEEE Trans. Autom. Control, vol. 41, no. 7, pp. 1041-1046, Jul. 1996.

[37] J. Shamma and M. Athans, "Analysis of gain scheduled control for nonlinear plants," IEEE Trans. Autom. Control, vol. 35, no. 8, pp. 898-907, Aug. 1990.

[38] A. Packard, "Gain scheduling via linear fractional transformations," Syst. Control Lett, vol. 22, pp. 79-92, 1994.

[39] A. Megretski and A. Rantzer, "System analysis via integral quadratic constraints," IEEE Trans. Autom. Control, vol. 42, no. 6 , pp. 819-830, Jun. 1997.

[40] P. A. Parrilo, "Structured semidefinite programs and semialgebraic geometry methods in robustness and optimization" Ph.D. dissertation, Caltech, Pasadena, CA, 2000 [Online]. Available: http://www. control.ethz.ch/-parrilo/pubs/

[41] K. G. Murty and S. N. Kabadi, "Some NP-complete problems in quadratic and nonlinear programming," Math. Program., vol. 39, pp. 117-129, 1987.

[42] P. A. Parrilo and B. Sturmfels, "Minimizing polynomial functions," in Workshop Algorithmic and Quantitative Aspects of Real Algebraic Geometry in Mathematics and Computer Science 2001, pp. 83-99.

[43] S. Prajna, A. Papachristodoulou, P. Seller, and P. A. Parrilo, "Sostools and its control applications," in Positive Polynomials in Control. New York: Springer-Verlag, 2005, pp. 273-292 [Online]. Available: http://www.cds.caltech.edu/sostools, http://www. mit.edu/parrilo/sostools

[44] L. Dai, Singular Control Systems. New York: Springer-Verlag, 1989.

[45] V. A. Yakubovic, "S-procedure in nonlinear control theory," Transl.: English Vestnik Leningrad University, vol. 4, no. 1, pp. 73-93, 1977, original Russian publication in Vestnik Leningradskogo Universiteta, Seriya Matematika, Leningrad, Russia, 1971, pp. 62-77.

[46] A. Papachristodoulou and S. Prajna, "Analysis of nonpolynomial systems using the sum of squares decomposition," in Positive Polynomials in Control, D. Henrion and A. Garulli, Eds. New York: Springer-Verlag, 2005, ch. 3, pp. 23-44.

[47] R. S. Smith and J. C. Doyle, "Model validation: A connection between robust control and identification," IEEE Trans. Autom. Control, vol. 37, no. 7, pp. 942-952, Jul. 1992.

[48] K. Poolla, P. Khargonekar, A. Tikku, J. Krause, and K. Nagpal, "A time-domain approach to model validation," IEEE Trans. Autom. Control, vol. 39, no. 5, pp. 951-959, May 1994.

[49] G. Dullerud and R. Smith, "A nonlinear functional approach to LFT model validation," Syst. Control Lett., vol. 47, no. 1, pp. 1-11, 2002.

[50] S. L. Campbell, Singular Systems of Differential Equations. Boston, MA: Pitman, 1980.

[51] A. van der Schaft and H. Schumacher, An Introduction to Hybrid Dynamical Systems. London, U.K.: Springer-Verlag, 2000.

[52] S. Prajna, A. Jadbabaie, and G. J. Pappas, "Stochastic safety verification using barrier certificates," in Proc. IEEE Conf. Decision and Control 2004, vol. 1, pp. 929-934.

[53] _ "A framework for worst case and stochastic safety verification using barrier certificates," IEEE Trans. Autom. Control, 2005, submitted for publication.

[54] B. Øksendal, Stochastic Differential Equations: An Introduction With Applications. Berlin, Germany: Springer-Verlag, 2000.

[55] H. J. Kushner, Stochastic Stability and Control. New York: Academic, 1967.
[56] B. Alberts et al., Molecular Biology of the Cell. New York: Garland, 2002.

[57] D. Straus, W. Walter, and C. Gross, “dnak, dnaj, and grpe heat shock proteins negatively regulate heat shock gene expression by controlling the synthesis and stability of $\sigma^{32}$," Genes Develop., vol. 4, no. 12A, pp. 2202-2209, 1990.

[58] M. Morita et al., "Translational induction of heat shock transcription factor $\sigma^{32}$ : Evidence for a built-in rna thermosensor," Genes Develop., vol. 13, no. 6, pp. 655-665, 1999.

[59] H. El-Samad, M. Khammash, H. Kurata, and J. Doyle, "Robustness analysis of the heat shock response in E. coli," in Proc. Amer. Control Conf. 2002, pp. 1742-1747.

[60] H. Kurata, H. El-Samad, T. Yi, M. Khammash, and J. Doyle, "Feedback regulation of the heat shock response in E. coli," in Proc. 40th IEEE Conf. Decision and Control 2001, pp. 837-842.

[61] M. Shea and G. Ackers, "The or control system in bacteriophage lambda: A physical-chemical model for gene regulation," J. Mol. Biol., pp. 211-230, 1985.

[62] J. Hasty, J. Pradines, M. Dolnik, and J. Collins, "Noise-based switches and amplifiers for gene expression," in Proc. Nat. Acad. Sci. USA 2000, vol. 97, pp. 2075-2080.

[63] C. Rao and A. Arkin, "Stochastic chemical kinetics and the quasisteady-state assumption: Application to the gillespie algorithm," $J$ Chem. Phys., vol. 118, pp. 4999-5010, 2003.

[64] D. Gillespie, "The chemical Langevin equation," J. Chem. Phys, vol. 113 , pp. 297-306, 2000

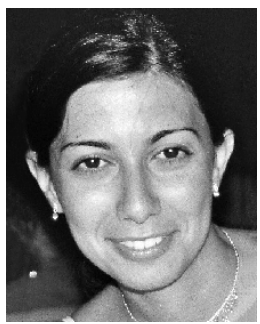

Hana El-Samad (Member, IEEE) received the B.E.E. degree from the American University, Beirut, Lebanon, in 1998, the M.S.E.E. from Iowa State University, Ames, in 1999, and the $\mathrm{Ph} . \mathrm{D}$. degree in mechanical engineering from the University of California, Santa Barbara, in 2004

In 2001, she was a Visiting Student at the California Institute of Technology, and in 2004 a Visiting Student at the University of California, San Francisco. She is currently a Faculty Fellow in the Biochemistry/Biophysics Department and the California Institute for Quantitative Biomedical Research, University of California, San Francisco. Her main research interests include applications of control theory and dynamical systems in physiology and molecular biology, stochastic dynamics, multiscale analysis, and noise-induced dynamical behavior in biological systems.

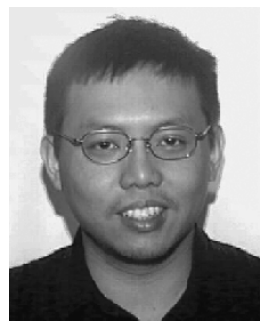

Stephen Prajna (Member, IEEE) received the B.Eng. degree in electrical engineering from the Bandung Institute of Technology, Indonesia, in 1998, the M.S. degree in mathematical sciences from the University of Twente, The Netherlands, in 2000, and the Ph.D. degree in control and dynamical systems from the California Institute of Technology, Pasadena, in 2005.

He has held short-term visiting positions at ETH Zurich, the Lund Institute of Technology, and the University of Pennsylvania. He is currently a Postdoctoral Scholar at the California Institute of Technology. His research interests include the general areas of systems and control, optimization, and their applications.

Dr. Prajna was a finalist in the Best Student Paper competition at the 2004 IEEE Conference on Decision and Control.

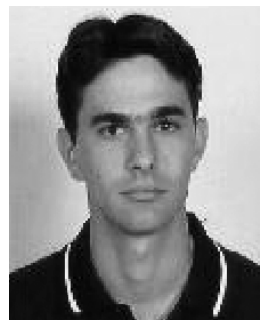

Antonis Papachristodoulou (Member, IEEE) was born in Limassol, Cyprus. He received the B.A.(M.A.)/M.Eng. degree in electrical and information sciences from the University of Cambridge, U.K., as a member of Robinson College in 2000 and the Ph.D. degree in control and dynamical systems with a minor in aeronautics from the California Institute of Technology, Pasadena, in 2005.

In Summer 2005, he visited the University of Cambridge, having received a David Crighton 
Fellowship. He is currently a Postdoctoral Scholar at the California Institute of Technology, and he will join the Department of Engineering Science at the University of Oxford, Oxford, U.K., in 2006 as a Departmental Lecturer in Control. His primary research interests are in scalable analysis of nonlinear systems using convex optimization based on sum of squares programming, and analysis and design of large-scale networked control systems with delays.

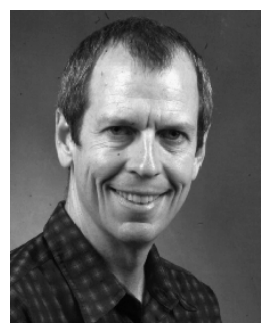

John Doyle (Member, IEEE) received the B.S. and M.S. degrees in electrical engineering from the Massachusetts Institute of Technology, Cambridge, in 1977, and the Ph.D. degree in mathematics from the University of California at Berkeley in 1984

He is the John G. Braun Professor of Control and Dynamical Systems, Electrical Engineering, and Bioengineering at the California Institute of Technology, Pasadena. His group led the development of the open source Systems Biology Markup Language (SBML) and the Systems Biology Workbench (SBW) ([Online]. Available: http://www.sbml.org), and also released the analysis toolbox SOSTOOLS ([Online]. Available: http://www.sbml.org). He was the theoretical lead on the team that developed the FAST protocol and shattered multiple world land speed records ([Online]. Available: http://netlab.caltech.edu). He has coauthored books and software toolboxes currently used at over 1000 sites worldwide, the main control analysis tool for high-performance commercial and military aerospace systems, as well as many other industrial systems. Early example industrial applications include X-29, F-16XL, F-15 SMTP, B-1, B-2, 757, Shuttle Orbiter, electric power generation, distillation, catalytic reactors, backhoe slope-finishing, active suspension, and CD players. His early work was in the mathematics of robust control, LQG robustness, (structured) singular value analysis, $\mathrm{H}$-infinity, plus recent extensions. His current research interests are in theoretical foundations for complex networks in engineering, biology, and multiscale physics.
Dr. Doyle received the 2004 IEEE Control Systems Award, the 1984 IEEE Centennial Outstanding Engineer Award, the 1984 Bernard Friedman Award, the 1983 American Automatic Control Council (AACC) Eckman Award, and the 1976 IEEE Hickernell Award. His best paper awards include the 1991 IEEE W. R. G. Baker Prize, the 1994 AACC O. Hugo Schuck Award, and the 1990 IEEE G. S. Axelby Award (twice). He has held national and world records and championships in various sports.

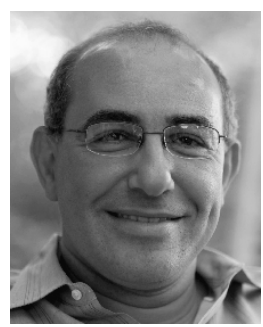

Mustafa Khammash (Senior Member, IEEE) received the B.S. degree in electrical engineering from Texas A\&M University, College Station, in 1986, and the Ph.D. degree in electrical engineering from Rice University, Houston, TX, in 1990 .

In 1990, he joined the Electrical Engineering Department at Iowa State University, Ames. While there, he created the dynamics and control program and led that control group until 2002 In 2001, he was a Visiting Professor at the California Institute of Technology, Pasadena. In May 2002, he joined the dynamics and control group in the Department of Mechanical and Environmental Engineering, University of California, Santa Barbara (UCSB). $\mathrm{He}$ is currently the Director of the Center for Control, Dynamical Systems, and Computations (CCDC) at UCSB. He also holds an appointment as Professor and Vice Chair of the Mechanical Engineering at UCSB. His research interests lie in the general area of dynamics and feedback control and its applications in natural and man-made systems. He works in the areas of robust control theory, multiobjective control, and systems biology.

Prof. Khammash is the recipient of the National Science Foundation Young Investigator Award, the Japan Society for the Promotion of Science (JSPS) Fellowship, the Iowa State University Foundation Early Achievement in Research and Scholarship Award, the ISU College of Engineering Young Faculty Research Award, and the Ralph Budd Best Engineering Ph.D. Thesis Award. 